\title{
Ultrafast base rotations mediate a solvent-assisted back-electron transfer in UV-excited DNA single strands
}

\author{
Benjamin Bauer ${ }^{1}$, Rahul Sharma ${ }^{2},{\text { Majed } \text { Chergui }^{1} \text {, and Malte Oppermann }}^{\star 1}$ \\ ${ }^{1}$ Laboratoire de Spectroscopie Ultrarapide and LACUS, École Polytechnique Fédérale de Lausanne, ISIC-FSB, \\ $\mathrm{CH}-1015$ Lausanne, Switzerland \\ ${ }^{2}$ Laboratory for Computation and Visualization in Mathematics and Mechanics, École Polytechnique Fédérale de \\ Lausanne, MATH-FSB, CH-1015 Lausanne, Switzerland \\ *malte.oppermann@epfl.ch
}

\section{ABSTRACT}

The photochemistry of DNA systems is characterized by the ultraviolet (UV) absorption of $\pi$-stacked nucleobases, resulting in exciton states delocalized over several bases. As their relaxation sensitively depends on local stacking conformations, disentangling the ensuing electronic and structural dynamics has remained an experimental challenge, despite their fundamental role in protecting the genome from potentially harmful UV radiation. Here we use transient absorption and transient absorption anisotropy spectroscopy with broadband femtosecond deep-UV pulses $(250-360 \mathrm{~nm})$ to resolve the exciton dynamics of UV-excited adenosine single strands under physiological conditions. Due to the exceptional deep-UV bandwidth and polarization sensitivity of our experimental approach, we simultaneously resolve the population dynamics, charge-transfer (CT) character and conformational changes encoded in the UV transition dipoles of the $\pi$-stacked nucleotides. Whilst UV excitation forms fully charge-separated CT excitons in less than $0.3 \mathrm{ps}$, we find that most decay back to the ground state via a solvent-assisted back-electron transfer. This deactivation mechanism is accompanied by a structural relaxation of the photoexcited base-stack, which we identify as an inter-base rotation of the nucleotides. Our results finally complete the exciton relaxation mechanism for adenosine single strands and offer a direct view into the coupling of electronic and structural dynamics in aggregated photochemical systems.

\section{Introduction}

The photochemical functions of natural and synthetic molecular aggregates are characterized by so-called exciton states that result from the electrostatic interactions of their constituent chromophores ${ }^{1,2}$. An important example are DNA systems, where the exciton transitions of $\pi$-stacked nucleobases dominate the absorption cross-section in the near ultraviolet (UV) region and thus play a crucial role in the formation of harmful photolesions and radical species, which may lead to skin cancer for example ${ }^{3}$. Indeed, whilst isolated nucleobase monomers efficiently dissipate their excitation energy on a sub-picosecond time-scale, single- (ssDNA) and double-stranded (dsDNA) DNA oligomers display excited state species with lifetimes 
up to the millisecond time scale ${ }^{4,5}$. However, whilst the initial exciton formation and ensuing charge separation processes across $\pi$-stacked bases have been studied in various model systems, achieving a complete picture of the exciton relaxation dynamics has remained challenging, because of the complex ground and excited state interactions between multiple bases in solution-phase DNA.

Due to the close proximity of $\pi$-stacked bases in B-form DNA $(\approx 3.4 \AA)$, the resulting electrostatic interactions generally comprise contributions from dipole-dipole, orbital overlap and electron exchange interactions $^{6}$. Consequently, the exciton states are characterized by two attributes ${ }^{7}$ : a delocalization length corresponding to the number of coupled bases and the degree of charge separation or charge-transfer (CT) character across them ${ }^{1}$. Importantly, the excitonic interactions sensitively depend on the geometrical arrangement of the coupled bases, such that the initially excited excitons are mostly determined by the ground state conformational ensemble. Their relaxation dynamics, however, crucially depend on the interbase coupling interactions in the photo-excited state and may therefore also involve local conformational changes of the oligomer ${ }^{8}$.

To this end, deoxyadenosine monophosphate ssDNA (denoted as $\mathrm{dA}_{\mathrm{n}}$ with $\mathrm{n}$ nucleotides) has emerged as a convenient helical model system with high stacking ratios of around $80 \%$ at room-temperature ${ }^{9}$. The monomer $\left(\mathrm{dA}_{1}\right)$ in the gas phase displays three close-lying lowest-energy transitions consisting of two optically bright ${ }^{1} \pi \pi^{*}$ transitions, $\mathrm{L}_{\mathrm{a}}$ and $\mathrm{L}_{\mathrm{b}}$, and an optically dark ${ }^{1} \mathrm{n} \pi^{*}$ transition ${ }^{10}$. In aqueous solution, $\mathrm{L}_{\mathrm{a}}$ is strongly stabilized, becoming the lowest energy LUMO singlet state, which also carries most of the oscillator strength ${ }^{11}$. From the Franck-Condon region, nearly barrierless pathways lead to conical intersections with the ground state surface, which rapidly deactivate the excited state on the sub-picosecond time scale forming a vibrationally hot ground state ${ }^{12}$. In ssDNA, excitonic interactions between stacked bases cause a prominent decrease in oscillator strength (hypochromism) and a slight blue-shift (hypsochromism) of the ${ }^{1} \pi \pi^{*}$ band maximum compared to the non-aggregated bases. These features are consistent with those of self-assembled H-aggregates of $\pi$-conjugated organic molecules ${ }^{13,14}$. However, even though these effects are well known since the $1960 \mathrm{~s}^{15,16}$, the exact delocalization length, CT character and energetic positions of the exciton states contributing to the static absorption of DNA

\footnotetext{
${ }^{1}$ Owing to Kasha's original definition, some authors have used the term 'exciton' to exclusively denote exciton states without any CT character. We explicitly include the possibility of a non-zero CT character in our use of the term, due to the presence of significant orbital overlap and electron exchange interactions in DNA systems.
} 
oligomers remain somewhat controversial. In $d A_{n}, L_{a}$ and $L_{b}$ transitions on different bases may couple and several theoretical treatments have predicted significant contributions from excitons delocalized over more than two stacked bases ${ }^{17-20}$. Nevertheless, strand-length dependent circular dichroism (CD) measurements, which are directly sensitive to dipolar couplings in the ground state, show that nearestneighbor interactions strongly dominate in the ${ }^{1} \pi \pi^{*}$ band ${ }^{21}$. In fact, there is growing consensus that the lowest-energy absorption band in $\mathrm{dA}_{\mathrm{n}}$ contains two exciton species: a neutral two-base exciton with zero CT character carrying most of the oscillator strength and a two-base CT exciton with nonzero CT character and a much weaker absorption coefficient ${ }^{8,19,22}$. Strong solvent interactions are expected to inhomogeneously broaden and red-shift the absorption band of the CT exction state and it is thus assumed to be the lowest-energy excited state, causing the enhanced low-energy absorption of $\mathrm{dA}_{\mathbf{n}}$ above $275 \mathrm{~nm}$, compared to monomeric $\mathrm{dA}_{1}{ }^{23,24}$.

Naturally, ultrafast studies on ssDNA have been instrumental in mapping out the exciton dynamics, albeit with often conflicting interpretations. Whilst the first optical experiments suggested excitons to be formed dynamically from photo-excited monomers ${ }^{25}$, this was later ruled out in favour of a direct excitation process. Here, early suggestions of average delocalization lengths of up to 4 bases ${ }^{26}$ were contested by mounting evidence for the exclusive excitation of two-base excitons, obtained from singlewavelength transient absorption (TA $)^{27,28}$ and time-resolved fluorescence experiments ${ }^{22}$. These studies broadly agreed on a mechanism where optically bright neutral 2-base excitons are excited first, followed by an ultrafast internal conversion to the optically dark minimum of the exciton band. These states would then acquire a CT character on the picosecond scale, possibly enabled by a reduction of the inter-base distance. Recently, Borrego-Varillas and co-workers were able to measure the upper limits of the involved time scales, finding that internal conversion to the neutral exciton band minimum completed in $100 \mathrm{fs}$, followed by a full charge-separation associated with an adenosine radical-ion pair in at most $3 \mathrm{ps}^{29}$.

In a parallel development, the vibrational marker bands of nucleotide radical-ions were identified and tracked in a series of ultrafast spectroscopy studies on UV-excited ssDNA model systems ${ }^{30,31}$. This corroborated the central role of CT excitons in the relaxation dynamics of ssDNA as the longest-living species dominating the dynamics beyond the first 10 ps. In addition, it has been established that a relevant fraction of the radical ions formed in adenosine stacks in both ss- and dsDNA survive well into the 
millisecond timescale, indicating that UV-excitation may provide an important contribution to the oxidative damage of $\mathrm{DNA}^{32,33}$. However, whilst the role of CT excitons as gateway states to DNA photodamage is now mostly settled, several aspects of their dynamic evolution have remained open. Importantly, a specific deactivation mechanism has not yet been established for $\mathrm{dA}_{\mathbf{n}}$. To this end, several possibilities have been considered, most notably a charge recombination process either via back-electron transfer ${ }^{19}$ or intra-strand proton-transfer ${ }^{34}$, a decay to a monomer-like state followed by internal conversion to the ground state ${ }^{35}$, and the dynamic formation of a bonded 2-base exciton species with a strongly reduced inter-base distance ${ }^{36}$. Even though such excited state conformational changes have been theoretically predicted to play an important role in their relaxation ${ }^{19,22,34,37}$, their direct observation has remained an experimental challenge, due to the current limitations of established spectroscopic techniques with combined time and structural resolution in the solution phase. Especially in the case of subtle motions of local chromophores or side chains, time-resolved X-Ray solution scattering does currently not provide the required spatial resolution ${ }^{38}$, whereas nuclear magnetic resonance spectroscopy does not provide the necessary time resolution ${ }^{39}$.

Here we pursue an alternative approach and employ a polarization-controlled pump-probe setup (see Methods) with femtosecond deep-UV probe pulses $(250-360 \mathrm{~nm})$ to conduct the first ultrafast spectroscopy experiments with broadband coverage of the ${ }^{1} \pi \pi^{*}$ band of $\mathrm{dA}_{20}<300 \mathrm{~nm}$. By combining transient absorption (TA) with transient absorption anisotropy (TAA) measurements, we simultaneously resolve the population dynamics, CT character and conformational changes encoded in the coupled UV transition dipoles of the photo-excited excitons. By modulating the strand-length, excitation wavelength and solvent environment we are then able to develop a complete reaction scheme. Quite remarkably, we find that CT excitons are already formed within the first 0.3 ps from initially excited neutral 2-base excitons, excluding any conformational dynamics in the charge-separation process. However, the transient anisotropy data suggests that ultrafast inter-base rotations drive the CT exciton to its minimum energy configuration in about 40 ps. Finally, by comparing the exciton dynamics in $\mathrm{H}_{2} \mathrm{O}$ and $\mathrm{D}_{2} \mathrm{O}$ buffer solution, we are able to provide conclusive evidence for a solvent-assisted back-electron transfer as the deactivation mechanism of the structurally relaxed CT excitons.

To establish these findings, we compare a large number of investigated sample systems, measurement 
conditions and experimental techniques. For achieving a clear presentation of the results without losing track of the discussion, we present and interpret each experiment in a separate section. We begin with the TA measurements of $\mathrm{dA}_{20}$ in $\mathrm{H}_{2} \mathrm{O}$ buffer solution and compare the kinetics observed for $266 \mathrm{~nm}$ and $285 \mathrm{~nm}$ excitation, thereby resolving different exciton formation mechanisms. Furthermore, a direct comparison with the TA kinetics of $\mathrm{dA}_{1}$ and $\mathrm{dA}_{2}$ highlights the close packing of bases in $\mathrm{dA}_{20}$ as a crucial factor in determining the exciton dynamics. In the following section we present molecular dynamics simulations to estimate the dipolar coupling strength between stacked bases in $\mathrm{dA}_{20}$, thereby establishing a link between its conformational ensemble and the photoexcited exciton states. Moving forward we present the TA measurements of the samples in $\mathrm{D}_{2} \mathrm{O}$ buffer solution, where the observed kinetic isotope effects suggest a solvent-assisted back-electron transfer as the primary exciton relaxation mechanism. The TAA measurements in $\mathrm{H}_{2} \mathrm{O}$ and $\mathrm{D}_{2} \mathrm{O}$ buffer solutions are presented in the final section and corroborate this mechanism, whilst also providing insights into the structural dynamics that accompany the exciton decay. Finally, we combine the above findings to establish a complete reaction scheme in the concluding section.

\section{Results and discussion}

\section{Transient absorption in $\mathrm{H}_{2} \mathrm{O}$ buffer solution}

This section compares three samples of $\mathrm{dA}_{\mathrm{n}}$ in aqueous phosphate buffer solution with different strand lengths (for sample preparation see Methods): the monomer $\mathrm{dA}_{1}$, the $\operatorname{dimer} \mathrm{dA}_{2}$, and the multimer $\mathrm{dA}_{20}$. Figure S.1 in the supporting information (SI) displays their steady-state absorption spectra, which are normalized to the respective band maxima to highlight differences in the band shapes. Compared to $\mathrm{dA}_{1}$, $\mathrm{dA}_{20}$ shows a pronounced blue-shift of the band maximum which can be attributed to the dipole-dipole interaction between stacked bases resulting in neutral exciton states. Below $275 \mathrm{~nm}, \mathrm{dA}_{20}$ displays an enhanced absorption, which has been attributed to a CT exciton band ${ }^{8,24}$. Whilst photoexcitation at 266 $\mathrm{nm}$ thus mainly populates neutral exciton states, photoexcitation at $285 \mathrm{~nm}$ is expected to predominantly excite CT excitons. Also note that whilst $\mathrm{dA}_{2}$ displays qualitatively similar spectral differences with $\mathrm{dA}_{1}$, these features are much less pronounced compared to $\mathrm{dA}_{20}$.

Fig. 1 displays normalized time- and wavelength-resolved TA maps for the three investigated samples with the UV-excitation wavelength indicated in brackets: $\mathrm{dA}_{1}(266 \mathrm{~nm}), \mathrm{dA}_{20}(266 \mathrm{~nm}), \mathrm{dA}_{20}(285 \mathrm{~nm})$, 

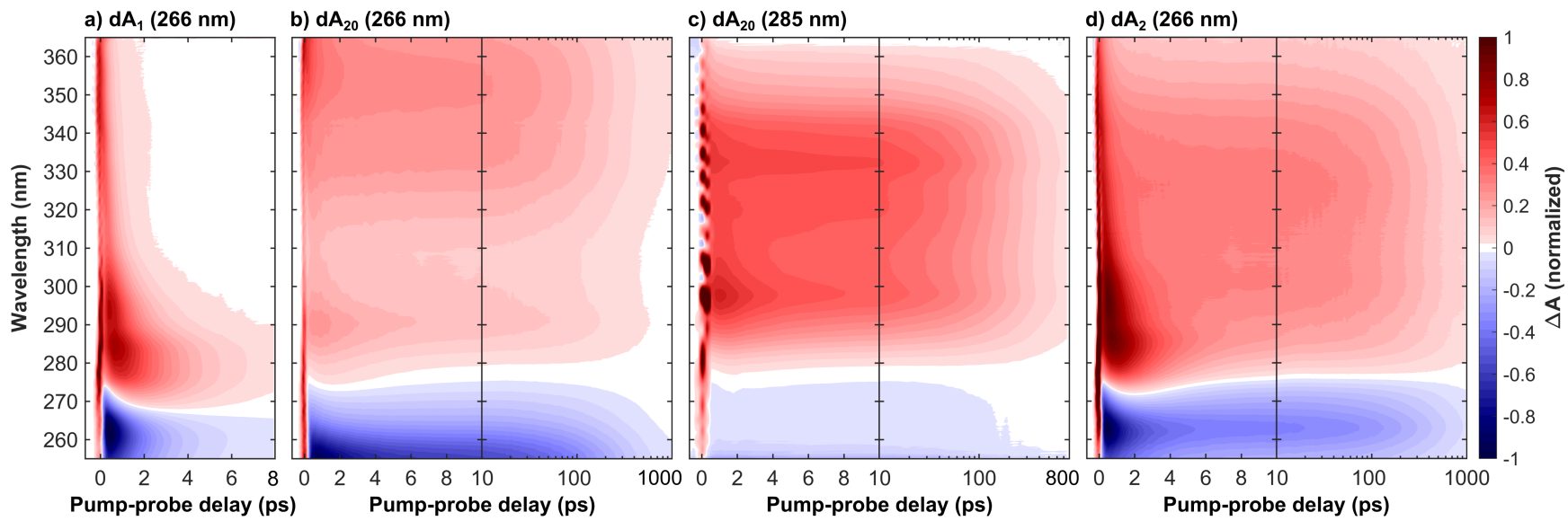

Figure 1. Transient absorption time-wavelength maps of a deoxyadenosine monophosphate ssDNA with $\mathrm{n}$ nucleotides in acqueous phosphate buffer solution and UV-excitation wavelength as indicated in brackets: a) Monomer $\mathrm{dA}_{1}(266 \mathrm{~nm})$, b) 20 -mer dA $20(266 \mathrm{~nm})$, c) 20 -mer $\mathrm{dA}_{20}(285 \mathrm{~nm})$, and d) dimer $\mathrm{dA}_{2}(266 \mathrm{~nm})$. The experimental time-resolution was $<300 \mathrm{fs}$ and maps (a,b,d) are normalized to the ground state bleach minimum near $266 \mathrm{~nm}$, whereas as map (c) was scaled to $70 \%$ of its exited state absorption maximum near $300 \mathrm{~nm}$ for improved comparability. Whilst the monomer's excited state absorption (ESA, positive signal) decays in $<10 \mathrm{ps}$, the multimer systems's ESA band decays on the 100 ps time scale and is associated with a CT exciton state. Their different band shapes $>10$ ps indicate that the formed CT exciton depends on both the excitation wavelength and strand length. A global analysis of the data assigns a $190 \mathrm{ps}$ time constant to its decay in $\mathrm{dA}_{20}$, independent of the excitation wavelength, whereas a much longer time constant of $340 \mathrm{ps}$ is observed in $\mathrm{dA}_{2}$. 
and $\mathrm{dA}_{2}(266 \mathrm{~nm})$. The original data sets are displayed in fig. S.4 and S.9 in the SI. All four TA maps display a negative TA amplitude $<275 \mathrm{~nm}$, associated with a ground state bleach (GSB), and a positive amplitude $>280 \mathrm{~nm}$, associated mostly with an excited state absorption (ESA). The direct comparison between the samples reveals striking differences in the GSB and ESA band shapes and decay kinetics. Whilst $\mathrm{dA}_{1}$ displays a narrow ESA maximum near $290 \mathrm{~nm}$ that decays on a picosecond timescale, the oligomer samples display a broad ESA band that decays two orders of magnitude more slowly. Because of the picosecond kinetics, we can identify these ESA bands with the long-lived CT exciton states that are exclusively formed in the oligomer systems. However, different ESA bandwidths are observed for the oligomer samples, which suggests that the precise nature and evolution of the formed CT excitons strongly depend on both the excitation wavelength and the number of nucleotides in the strand. In the following we will first focus on the TA data for pump-probe delays $<1$ ps to discuss the CT exciton formation mechanism. We will then focus on the TA data for pump-probe delays $>1$ ps to discuss their relaxation mechanism.

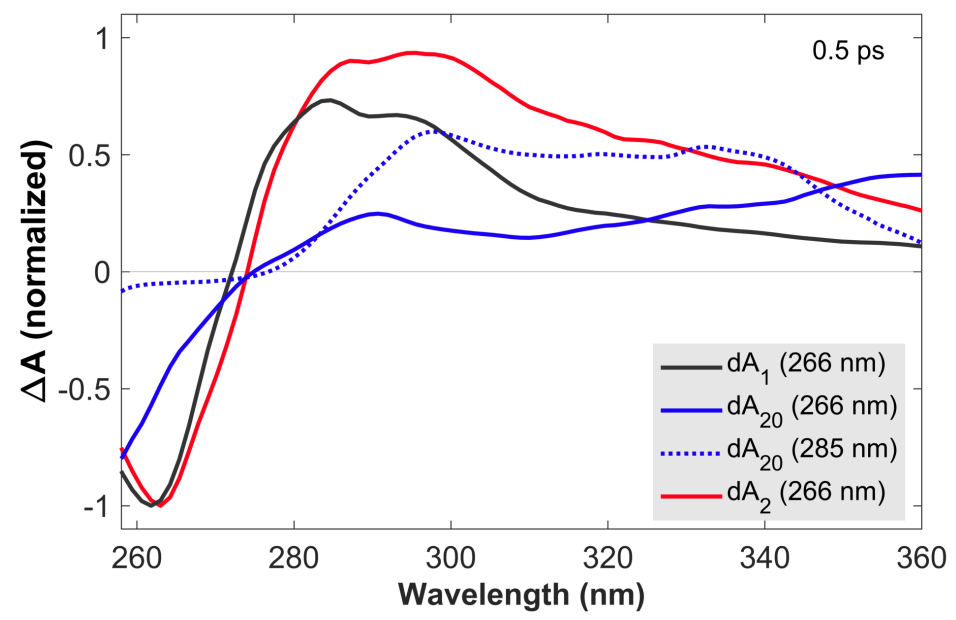

Figure 2. TA spectra of $\mathrm{dA}_{1}, \mathrm{dA}_{20}$, and $\mathrm{dA}_{2}$ in aqueous phosphate buffer solution at a pump-probe delay of 0.5 ps. All samples were photoexcited at $266 \mathrm{~nm}$, whereas $\mathrm{dA}_{20}$ was also excited at $285 \mathrm{~nm}$, as indicated. For $266 \mathrm{~nm}$ excitation, the TA spectra are normalized to the GSB minimum near $265 \mathrm{~nm}$, whereas the normalization factor for $\mathrm{dA}_{20}$ was adapted in accordance with the curvature of this feature. The TA spectrum for $\mathrm{dA}_{20}(285 \mathrm{~nm})$ was scaled to $70 \%$ of the ESA maximum. The comparison of the GSB bands suggests that whilst $\mathrm{dA}_{2}$ is dominated by monomeric excitations, these are mostly absent in $\mathrm{dA}_{20}$, where excitonic transitions dominate. Comparing $\mathrm{dA}_{20}(266 \mathrm{~nm})$ and $\mathrm{dA}_{20}(285 \mathrm{~nm})$ shows that two different exciton species are excited, resulting in strong spectral differences in the GSB and ESA bands.

To this end, fig. 2 compares the early-time TA spectra of the adenosine samples at a pump-probe 
delay of 0.5 ps. Whilst $\mathrm{dA}_{1}(266 \mathrm{~nm})$ and $\mathrm{dA}_{2}(266 \mathrm{~nm})$ display mostly identical GSB bands, $\mathrm{dA}_{20}$ $(266 \mathrm{~nm})$ shows a pronounced blue-shift of $>10 \mathrm{~nm}$. In comparison, the GSB band of dA $20(285 \mathrm{~nm})$ is extremely weak and likely extends further towards the mid-UV region. In the ESA region, $\mathrm{dA}_{1}$ and $\mathrm{dA}_{2}$ show relatively similar spectral shapes, albeit with different amplitudes, dominated by a pronounced band centred near $290 \mathrm{~nm}$ and a tail decaying towards $370 \mathrm{~nm}$. In contrast, dA $20(266 \mathrm{~nm})$ shows only a very weak ESA band at $290 \mathrm{~nm}$, whereas such a band is not observable in dA 20 (285 nm). Both 20-mers display a broad ESA band, however $\mathrm{dA}_{20}(266 \mathrm{~nm})$ has an additional ESA band near $370 \mathrm{~nm}$.

Starting with $\mathrm{dA}_{1}$, we can assign its GSB to the $\mathrm{L}_{\mathrm{a}}$ transition and the main ESA band to the hot ground state, consistent with the literature ${ }^{12}$. The tail extending into the near-UV has previously been assigned to the almost fully decayed $\mathrm{L}_{\mathrm{a}}$ state located near $370 \mathrm{~nm}^{40,41}$. The strongly blue-shifted GSB in dA $20(266$ $\mathrm{nm}$ ) is consistent with its blue-shifted steady state absorption spectrum and confirms that at $266 \mathrm{~nm}$, a direct photo-excitation channel to high-energy bright neutral excitons dominates. The corresponding ESA region therefore only shows a residual contribution from a hot monomeric ground state near $290 \mathrm{~nm}$, likely resulting from monomer-like excitations of unstacked nucleotides within the strand ${ }^{28}$. Instead, the ESA band is dominated by an additional maximum near $370 \mathrm{~nm}$, which decays rapidly on the sub-picosecond time scale (see fig. 1c). We thus assign this band to the neutral exciton ESA from coupled $\mathrm{L}_{\mathrm{a}}$ transition dipoles, which rapidly decays via internal conversion to the neutral exciton band minimum, consistent with recent theoretical and experimental studies ${ }^{29,40}$. In direct contrast, $\mathrm{dA}_{20}(285 \mathrm{~nm})$ does not display any of the spectral signatures of the neutral exciton or any monomeric excitation. However, its weak GSB band is consistent with the direct excitation of a (mostly) optically dark CT exciton with a strongly inhomogeneously broadened absorption band, as suggested in the literature. The observed ESA band can be attributed entirely to a CT exciton, which thus corresponds to the lowest-energy excited state in $\mathrm{dA}_{20}$. Finally, the GSB in $\mathrm{dA}_{2}$ suggests that $266 \mathrm{~nm}$ excitation mostly populates monomeric $\mathrm{L}_{\mathrm{a}}$ states without any clear evidence for the direct excitation of neutral or CT excitons. However, whilst the ESA is dominated by a hot monomeric ground state, the differences in ESA amplitudes between $\mathrm{dA}_{1}$ and $\mathrm{dA}_{2}$ suggest the presence of an additional broad ESA band that is not observed in the monomer.

We now proceed to the discussion of the TA data for pump-probe delays $>1 \mathrm{ps,} \mathrm{as} \mathrm{displayed} \mathrm{in} \mathrm{fig.} 1$. In $\mathrm{dA}_{1}$, the hot ground state's ESA feature decays on the picosecond timescale due vibrational cooling, 
consistent with the time-dependent blue-shift of its spectral weight and the simultaneous recovery of the ground state. In contrast, $\mathrm{dA}_{20}(266 \mathrm{~nm})$ shows a negligible contribution from this channel and instead displays an ESA decay in $>10$ ps. Following the work by Borrego-Varillas and co-workers ${ }^{29}$, the ESA band on this scale is due to the absorption of a two-base CT exciton, associated with a fully separated adenosine radical ion pair. This implies that the long-lived ESA band in $\mathrm{dA}_{20}(285 \mathrm{~nm})$ must describe a different CT exciton species, either with a different delocalization length or a reduced CT character. To this end, the comparison with $\mathrm{dA}_{2}$ offers further insights. Whilst the vibrational cooling of the hot monomeric ground state dominates the kinetics $<10$ ps, the ESA decay $>10$ ps is slower than in both experiments on $\mathrm{dA}_{20}$. Importantly, the long-lived ESA in $\mathrm{dA}_{2}$ takes a band shape that is remarkably similar to $\mathrm{dA}_{20}(285$ $\mathrm{nm})$. Indeed, in fig. S.9d in the SI, we show that the early-time TA spectrum of $\mathrm{dA}_{2}(266 \mathrm{~nm})$ can be very well reproduced by a sum of the scaled TA spectra of $\mathrm{dA}_{1}$ and $\mathrm{dA}_{20}(285 \mathrm{~nm})$ at the same pump-probe delay of 0.5 ps. We thus suggest that two-base CT exciton with a partial CT character is formed in both in $\mathrm{dA}_{2}$ and $\mathrm{dA}_{20}(285 \mathrm{~nm})$.

To quantify the TA kinetics, we performed global fits on each of the presented TA data sets. To this end, we employed a sequence of multi-exponential functions convoluted with a Gaussian instrument response function (for details see Methods and section S.3 in the SI). The resulting decay associated spectra (DAS) and residuals are displayed in fig. S.4 and S.9 in the SI and the obtained decay constants are displayed in table 1. Each decay constant is reported with an error of $10 \%$. The kinetics of $\mathrm{dA}_{1}$ can be accurately fitted with a minimum of two exponential functions, with time constants $\tau_{1}^{(1)}=0.7 \pm 0.1 \mathrm{ps}$ and $\tau_{2}^{(1)}=1.9 \pm 0.2 \mathrm{ps}$. Even though we obtain a high accuracy for the performed fits, it is well known that the spectral shifts caused by vibrational cooling dynamics cannot be modeled adequately by globally fitted multi-exponential functions ${ }^{42}$. We therefore neglect a detailed analysis of the short-time dynamics. Nevertheless, this has no impact on the fitting accuracy of the kinetics that are orders of magnitude slower and thus unaffected by the cooling dynamics. The fits for the remaining oligomers display similar kinetic components $<10$ ps, but notably require two additional slower decay components to achieve an adequate fit of the data: a time constant $\tau_{3}$ on the 100 ps scale, associated with the deactivation of the CT exciton, and $\tau_{4}$, which is much longer than the maximum pump-probe delay and is associated with a long-lived photo-product $^{32}$. First of all we note that the value obtained for $\tau_{3}^{(20,266)}=190 \pm 20$ agrees well with 
previously published data ${ }^{28}$. Remarkably, we find that in $\mathrm{dA}_{20}$ this decay constant is mostly independent of the excitation wavelength, as we obtain $\tau_{3}^{(20,285)}=180 \pm 20 \mathrm{ps}$ for $\mathrm{dA}_{20}(285 \mathrm{~nm})$. This is in contrast with the much longer decay constant $\tau_{3}^{(2)}=340 \pm 30$ ps obtained for $\mathrm{dA}_{2}(266 \mathrm{~nm})$. In addition, fig. S.9e compares the DAS for $\tau_{3}$ for the three oligomer samples and shows mostly identical spectral shapes for $\mathrm{dA}_{20}(285 \mathrm{~nm})$ and $\mathrm{dA}_{2}(266 \mathrm{~nm})$ in the ESA region, whereas the corresponding DAS for $\mathrm{dA}_{20}(266 \mathrm{~nm})$ extends further beyond $360 \mathrm{~nm}$.

On the basis of these observations, we thus propose two different excitation pathways in $\mathrm{dA}_{20}$ : (1) the dynamic formation of a fully charge-separated CT exciton via an optically bright neutral two-base exciton, and (2) the direct excitation of a CT exciton, which displays a reduced CT character at $285 \mathrm{~nm}$ excitation. Since the observed CT exciton decay time is independent of the formation pathway, there is likely a single $\mathrm{CT}$ exciton band in $\mathrm{dA}_{20}$, which can be accessed via an internal conversion process from the neutral exciton band. Note that whilst the absorption of the CT exciton band is sufficiently broad to be excited at $266 \mathrm{~nm}$ as well, its contribution to the total yield is expected to be negligible, due to its low oscillator strength. The same reasoning thus applies to $\mathrm{dA}_{2}$, even though an ESA signature of neutral excitons is not resolved in the TA data. This is surprising, since the non-zero CD spectrum of $\mathrm{dA}_{2}$ clearly shows the presence of dipolar couplings between the nucleobases ${ }^{35}$. However, we propose that the number of strongly coupled bases is much lower in $\mathrm{dA}_{2}$ than in $\mathrm{dA}_{20}$, because the greater steric freedom of $\mathrm{dA}_{2}$ is expected to lead to a much broader conformational ensemble. Since the dipolar coupling strength $J$ between neighbouring bases has an $R^{-3}$ dependence (with $R$ denoting the inter-base distance) and is maximised for parallel transition dipoles (see eq. 2 in Methods), the average dipolar coupling strength is thus expected to be reduced compared to $\mathrm{dA}_{20}$.

\section{Molecular dynamics simulations}

In order to rationalize the discussion of the TA data, we conducted molecular dynamics simulations of the ground state configurational ensemble of $\mathrm{dA}_{20}$ and $\mathrm{dA}_{2}$ (for computational details see Methods). Fig. 3 , displays the probability distribution of nearest-neighbour configurations as a function of their dipolar coupling strength, for three different dimer classes: (1) an isolated $\mathrm{dA}_{2},(2)$ the two-base stacks at the ends of a strand ( $\mathrm{dA}_{20}$ ends), and (3) the average of all two-base stacks in the strand ( $\mathrm{dA}_{20}$ mean). As 


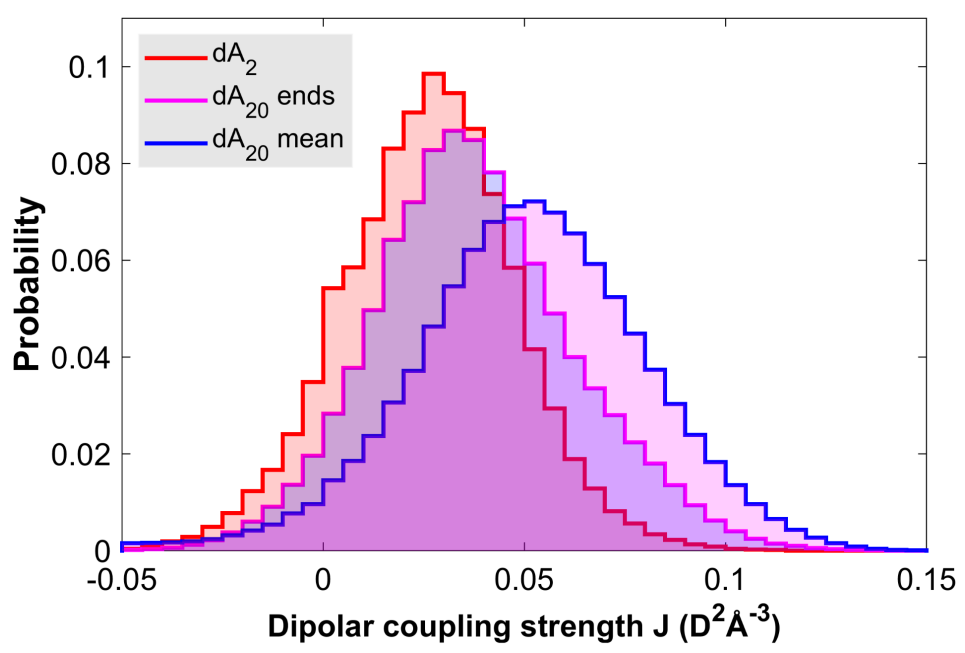

Figure 3. Molecular dynamics simulation of the ground state ensembles of solvated $\mathrm{dA}_{2}$ and $\mathrm{dA}_{20}$. The probability distributions are obtained by binning the nearest-neighbour base stack configurations according to their calculated dipolar coupling strength $J$ (see Methods) for an isolated dimer $\left(\mathrm{dA}_{2}\right)$, for the end-pairs in the strand ( $\mathrm{dA}_{20}$ ends) and for all base-pairs in the strand ( $\mathrm{dA}_{20}$ mean). The results show that the closer packing in $\mathrm{dA}_{20}$ leads to a significant increase of nearest-neighbour stacks with a strong dipolar coupling.

expected, the probability distribution for a free $\mathrm{dA}_{2}$ in solution is strongly shifted towards lower values of $J$ compared to the mean of dimer configurations within $\mathrm{dA}_{20}$, whilst also showing a much larger number of configurations with $J$ close to zero. Note that the probability distribution of the stacks at the end of the strand describes an intermediate case, since only one of the bases has two nearest neighbours. Even though this simulation is based on a simplified definition of the dipolar coupling strength, it strongly suggests that the close packing of nucleotides in $\mathrm{dA}_{20}$ reduces the conformational heterogeneity of inter-base geometries and thus increases the number of neutral exciton states at the expense of monomeric excited states. Similarly, we propose that electron exchange and orbital overlap interactions are increased, such that the $\mathrm{CT}$ exciton in $\mathrm{dA}_{20}(266 \mathrm{~nm})$ displays a larger $\mathrm{CT}$ character than in $\mathrm{dA}_{2}(266 \mathrm{~nm})$. In this view, the $\mathrm{CT}$ character of the formed exciton is determined by the base stack conformation upon photo-excitation. We thus speculate that the lowest energy CT exciton excited in $\mathrm{dA}_{20}(285 \mathrm{~nm})$ corresponds to a strongly solvent-stabilized conformation with an increased inter-base distance, for example.

\section{Transient absorption in $\mathrm{D}_{2} \mathrm{O}$ buffer solution}

Since both $\mathrm{dA}_{20}(266 \mathrm{~nm})$ and $\mathrm{dA}_{20}(285 \mathrm{~nm})$ display a significantly faster CT exciton decay time than $\mathrm{dA}_{2}$ $(266 \mathrm{~nm})$, it is likely that the close packing of $\mathrm{dA}_{20}$ also plays a role in its deactivation mechanism. This 
suggests a process that is sensitive to the spatial configuration of the base-stack, such as a solvent-assisted back-electron transfer and an intra-strand proton transfer, for example. We thus conducted TA experiments in heavy water $\left(\mathrm{D}_{2} \mathrm{O}\right)$ phosphate buffer solution to obtain further insights into the CT decay mechanism. Fig. S.6 in the SI displays the obtained TA data sets for $\mathrm{dA}_{1}(266 \mathrm{~nm}), \mathrm{dA}_{20}(266 \mathrm{~nm})$, and dA $2(266$ $\mathrm{nm}$ ) and the results of their global analysis. The obtained decay constants are reported in table 1 . Whilst the observed vibrational cooling dynamics are slightly slower as expected, a significant kinetic isotope effect (KIE) is observed for the CT exciton decay constant $\tau_{3}$. Defining $K=\tau_{3}^{\mathrm{D}} / \tau_{3}^{\mathrm{H}}=k_{3}^{\mathrm{H}} / k_{3}^{\mathrm{D}}$, we obtain moderate KIEs of $K^{(20)} \approx 1.7$ for $\mathrm{dA}_{20}(266 \mathrm{~nm})$ and $K^{(2)} \approx 2.1$ for $\mathrm{dA}_{2}(266 \mathrm{~nm})$. This is somewhat surprising, as previous studies on dsDNA have only detected KIEs for alternating base-sequences and no deuteration effects in non-alternating sequences ( were assigned to a sequential process with an initial intra-strand exction formation followed by quenching through inter-strand proton transfer ${ }^{45,46}$. Taking into account the lack of base-pairing interactions, we thus note that the values for $K^{(20)}$ and $K^{(2)}$ are consistent with KIEs commonly observed for a solvent-assisted back-electron transfer in radical-ion pairs ${ }^{47}$. Whilst an intra-strand proton transfer might not be excluded on this basis, the relatively large average inter-base distance of $\approx 3.4 \AA$ in B-form DNA is expected to lead to a high reaction barrier ${ }^{34}$ and a significantly higher $\mathrm{KIE}^{48}$.

In this view, it is important to consider two ways in which the solvent deuteration may alter the electronic properties of $\mathrm{dA}_{\mathrm{n}}$. First, hydrogen-bonding is a key factor in mediating base-stacking ${ }^{49}$, such that its weakening through deuteration may affect the ground state conformational ensemble and thus the excitonic coupling interactions. However, the $\mathrm{CD}$ spectra for $\mathrm{dA}_{20}$ and $\mathrm{dA}_{2}$ in $\mathrm{H}_{2} \mathrm{O}$ and $\mathrm{D}_{2} \mathrm{O}$ buffer solution (see fig. S.2) display nearly identical shapes, suggesting a negligible impact on the inter-base dipolar couplings. Second, it is well known that solvation in $\mathrm{D}_{2} \mathrm{O}$ buffer solution leads to deuteration of the amino group of $\mathrm{dA}_{1}{ }^{50}$. Here, solvent-dependent studies on structurally similar naphthylalkylamines have shown that amino-deuteration and the increase of its ionization potential through the weakened hydrogen-bonding network lead to a reduced CT character of the intra-molecular CT exciton state ${ }^{51}$. Similar arguments have also been employed by Mataga and coworkers to explain the solvent-dependent CT exciton decays of hydrogen-bonded molecular ion-pairs involving amino groups as proton donors ${ }^{52}$. 


\section{Transient absorption anisotropy in $\mathrm{H}_{2} \mathrm{O}$ and $\mathrm{D}_{2} \mathrm{O}$ buffer solution}

In transient absorption anisotropy (TAA), a linearly polarized excitation pulse creates a partially aligned ensemble of photoexcited molecules. Note that for a given angle $\theta$ between the excited transition dipole

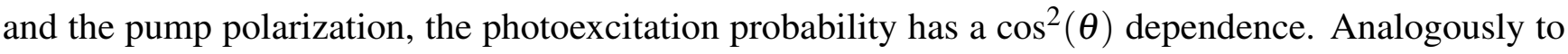
fluorescence anisotropy, TAA measures the TA difference of a parallel and perpendicular pump and probe pulse polarization configuration, according to eq. 1 in Methods. As a consequence, the TAA of the ensemble average takes a maximum value of $r=0.4$ if the probed transition dipole is parallel to the one initially excited and it takes a minimum value of $r=-0.2$ if they are perpendicular. In the GSB region, TAA probes the time-dependent orientation of the bleached transition dipoles with respect to the originally excited ground state transition dipole. In the ESA region, TAA then probes the relative orientation between the excited state transition dipole and the original photoexcitation axis within the molecule's frame of reference.

To this end, fig. S.10 displays time- and wavelength-resolved TAA maps of dA, $\mathrm{dA}_{20}(266 \mathrm{~nm})$, and $\mathrm{dA}_{20}(285 \mathrm{~nm})$ in $\mathrm{H}_{2} \mathrm{O}$ buffer solution, whereas fig. $\mathrm{S} .12$ displays the TAA maps of $\mathrm{dA}_{2}$ and $\mathrm{dA}_{20}(266$ $\mathrm{nm})$ in $\mathrm{D}_{2} \mathrm{O}$ buffer solution. All maps display positive TAA values in both the GSB and ESA bands, which decay to zero in a global fashion due to rotational diffusion. Note that due to its mathematical definition, the calculated TAA diverges to infinity when the TA signal approaches zero, which takes place both for zero-crossings between the GSB and ESA bands and for low excited state populations at long pump-probe delays. However, except near the zero-crossings, the TAA bands do not display any significant spectral dependence. The uniform ESA bands of $\mathrm{dA}_{2}$ and $\mathrm{dA}_{20}$ therefore provide further evidence that the CT exciton is the only excited species probed in this region ${ }^{53}$. As for the TA data, we first compare the spectral shapes of the TAA spectra at early pump-probe delays and then proceed to discuss the time-evolution of the TAA signal.

Fig. 4a displays the TAA spectra in the ESA region for a pump-probe delay of $3 \mathrm{ps,} \mathrm{where} \mathrm{CT} \mathrm{excitons}$ are expected to be fully formed and any ESA from a hot monomeric ground state mostly decayed. In $\mathrm{dA}_{20}(266 \mathrm{~nm})$ and $\mathrm{dA}_{2}(266 \mathrm{~nm})$, most CT excitons are formed through neutral excitons and thus the excitation of coupled $\mathrm{L}_{\mathrm{a}}$ transition dipoles within a base-stack. This implies a photoexcitation axis parallel to the nucleobase plane. As a charge separation across $\pi$-stacked bases creates a transition dipole moment 

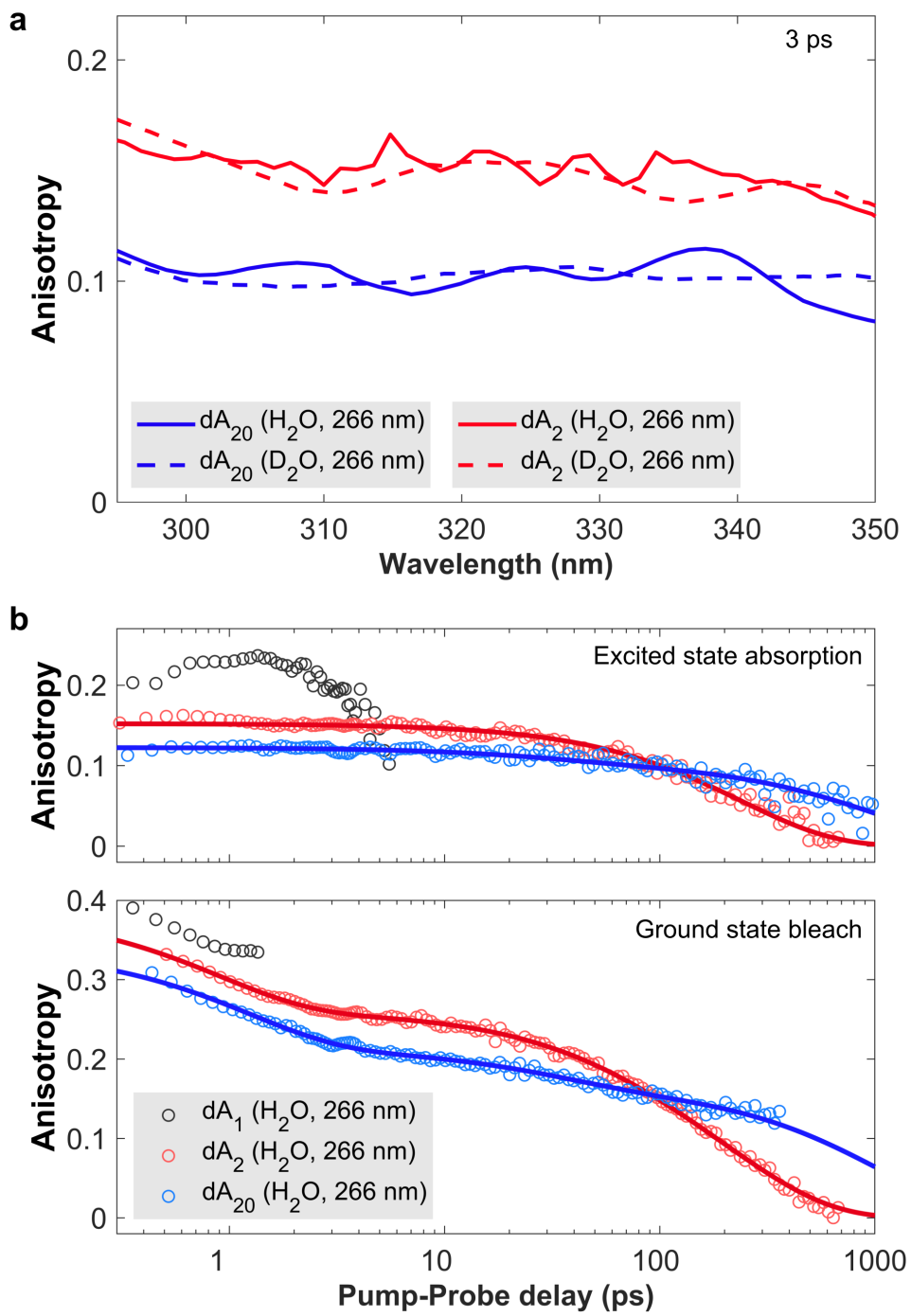

Figure 4. a)Transient absorption anisotropy (TAA) spectra in the ESA region of $\mathrm{dA}_{2}(266 \mathrm{~nm})$ and $\mathrm{dA}_{20}$ $(266 \mathrm{~nm})$ in $\mathrm{H}_{2} \mathrm{O}$ and $\mathrm{D}_{2} \mathrm{O}$ phosphate buffer solution at a pump-probe delay of $3 \mathrm{ps}$. In the ESA region, TAA probes the orientation of the transition dipole of the CT exciton and provides an indirect marker of its CT character. This implies that solvent deuteration has no impact on the CT character of the excitons, thus ruling out the involvement of the amino group as a proton donor. b)Transient absorption anisotropy (TAA) decay traces of deoxyadenosine monophosphate monomer $\left(\mathrm{dA}_{1}\right)$, dimer $\left(\mathrm{dA}_{2}\right)$ and a 20 -mer $\left(\mathrm{dA}_{20}\right)$ in phosphate buffer solution. The data points (open circles) are obtained by spectrally averaging the TAA over the GSB and ESA bands with the exact boundaries listed in the SI. Global fits of the spectrally resolved TAA data are presented in detail in the SI with representative curves included in the figure as solid lines. In the GSB region, TAA is sensitive to the distribution of bleached ground state transition dipoles and reveals a conformational change of the CT exciton in $\mathrm{dA}_{20}$ with a 40 ps time constant.

perpendicular to the nucleobase planes, any increase in CT character would lead to a decrease of the measured TAA. On this basis, the comparison of the TAA spectra confirms that the CT character of the CT exciton in $\mathrm{dA}_{20}(266 \mathrm{~nm})$ is indeed higher than in $\mathrm{dA}_{2}(266 \mathrm{~nm})$. Quite remarkably, however, changing the 
solvent from $\mathrm{H}_{2} \mathrm{O}$ to $\mathrm{D}_{2} \mathrm{O}$ has no appreciable effect on the measured TAA in any of the samples. Therefore, the amino group does not play a role in the CT exciton formation and we may rule out any contribution as a proton donor in an intra-strand proton transfer. We thus conclude that in $\mathrm{dA}_{\mathrm{n}}, \mathrm{CT}$ excitons are quenched via a solvent-assisted electron transfer. The observed KIEs then result from a reduced vibrational coupling upon solvent deuteration, whereas the longer CT exciton decay times in $\mathrm{dA}_{2}$ are likely caused by the larger inter-base distances within its ground state conformational ensemble.

We now proceed to discuss the time-evolution of the TAA data. To this end, fig. $4 \mathrm{~b}$ displays the TAA of $\mathrm{dA}_{1}, \mathrm{dA}_{2}$, and $\mathrm{dA}_{20}(266 \mathrm{~nm})$ as a function of pump-probe delay. The kinetic traces were obtained by averaging the TAA in the GSB and ESA regions with the exact spectral boundaries reported in table S.1 in the SI. Starting with the ESA region, the TAA probes the relative orientation between the photo-excited ground and the excited state transition dipoles. In $\mathrm{dA}_{1}$, this is the hot ground state, whereas in the two oligomers it is associated with the $\mathrm{CT}$ exciton. In $\mathrm{dA}_{1}$ the TAA reaches a maximum value of approximately 0.23 and then decays to zero, which we attribute to rotational diffusion. In $\mathrm{dA}_{2}$ the anisotropy takes a value of 0.15 at the earliest pump-probe delay of $0.3 \mathrm{ps}$ and decays mono-exponentially to zero on the 100 ps timescale. In direct comparison, $\mathrm{dA}_{20}$ displays a lower initial TAA value of about 0.12 and decays bi-exponentially to zero with a minor component on the $100 \mathrm{ps}$ timescale and a major component on the 1000 ps timescale. In each oligomer, the slowest decay component has to be attributed to rotational diffusion. In addition, two processes may change the orientation of the transition dipole of the CT exciton: a conformational change of the nucleobases in the stack and a change in CT character. Importantly, neither $\mathrm{dA}_{2}$ nor $\mathrm{dA}_{20}$ display any change in anisotropy during the first 3 ps. This implies, that neither a change in base stack conformation, nor in CT character are taking place on this time scale. Instead, we conclude that the CT character of the exciton is fully formed in $<0.3 \mathrm{ps,}$ which corresponds to the time-resolution of our setup (see fig. S.3 in the SI). This implies that CT exciton formation in ssDNA is unlikely to involve a conformational change of the base stack, such as a continuous approach of the bases, as has been suggested by several authors ${ }^{22,29}$. Instead, we propose that whilst some CT excitons may be formed directly upon photo-excitation, the majority are formed via an inter-base electron transfer on the sub-picosecond timescale, preceding any conformational change.

In the GSB region, TAA probes the ensemble of the bleached ground state ${ }^{1} \pi \pi^{*}$ transition dipoles 
that are parallel to the molecular plane of the adenosine nucleotide. As a benchmark case, we note that the TAA of $\mathrm{dA}_{1}$ takes an initial value close to 0.4 , as expected for a single bleached transition dipole. During the first 3 ps, all three samples experience a rapid TAA decay with a time constant denoted as $\rho_{1}$. For $\mathrm{dA}_{1}$, the TAA diverges beyond $2 \mathrm{ps}$, due to a low TA signal (see fig. S.10 in the SI). Just as in the ESA region the oligomers display additional decay components: whilst the TAA of dA 20 decays biexponentially with decay constants $\rho_{2}$ and $\rho_{3}, \mathrm{dA}_{2}$ decays monoexponentially. To extract the associated time constants, we performed global fits of the complete time-wavelength TAA maps instead of individual single-wavelength traces. As the probed spectral window generally contains contributions from different transition dipoles, the observed TAA kinetics may vary strongly as a function of probe wavelength. In addition, the divergence observed at zero-crossings between GSB and ESA bands and low TA signals may further distort the observed TAA kinetics, especially when analyzing only single-wavelength kinetic traces. To mitigate the described effects, we performed global fits of a sum of exponential decays, convoluted with the IRF. As described in detail in section S.4, probe wavelengths near zero-crossings and band edges were excluded, along with pump-probe delays associated with a low TA amplitude. For dA $20(266 \mathrm{~nm})$ and $\mathrm{dA}_{2}$ in $\mathrm{H}_{2} \mathrm{O}$ and $\mathrm{D}_{2} \mathrm{O}$, fig. S.10 and S.12 display the time-wavelength maps of the obtained residuals, whereas fig. S.10 and S.12 display the resulting DAS and representative kinetic traces from the GSB and ESA region. The obtained time constants are displayed in table 1. The individual errors were estimated by systematically varying the spectral boundaries and cut-offs in the pump-probe delays included in the fit and noting the impact on the fit results.

Starting with the TAA data obtained in $\mathrm{H}_{2} \mathrm{O}$ buffer solution, we note that the slowest decay constants $\rho_{3}^{(2)}$ and $\rho_{3}^{(20)}$ for $\mathrm{dA}_{2}$ and $\mathrm{dA}_{20}$, respectively, must denote their rotational diffusion times. To assign the fastest decay constant $\rho_{1}$, we observe that the associated DAS for $\mathrm{dA}_{2}$ and $\mathrm{dA}_{20}$ only displays nonzero values $<310 \mathrm{~nm}$, with a band shape that is consistent with the ESA of the hot ground state in $\mathrm{dA}_{1}$. Taking into account that an identical sub-picosecond decay is observed in $\mathrm{dA}_{1}$, we conclude that $\rho_{1}$ likely describes the depolarization of the hot monomeric ground state's transition dipole due to vibrational cooling. The contribution of $\rho_{1}$ to the TAA kinetics in the GSB region is thus likely caused by the overlapping monomeric ESA band.

The most interesting aspect of the TAA decay is the intermediate time constant $\rho_{2}^{(20)}=44 \pm 10$ ps 


\begin{tabular}{|l|l|l|l|l||l|l|l|}
\hline Sample & $\tau_{1}(\mathrm{ps})$ & $\tau_{2}(\mathrm{ps})$ & $\tau_{3}(\mathrm{ps})$ & $\tau_{4}(\mathrm{ps})$ & $\rho_{1}(\mathrm{ps})$ & $\rho_{2}(\mathrm{ps})$ & $\rho_{3}(\mathrm{ps})$ \\
\hline $\mathrm{dA}_{1}, \mathrm{H}_{2} \mathrm{O}, 266 \mathrm{~nm}$ & $0.7 \pm 0.1$ & $2.0 \pm 0.2$ & - & - & - & - & - \\
\hline $\mathrm{dA}_{2}, \mathrm{H}_{2} \mathrm{O}, 266 \mathrm{~nm}$ & $0.7 \pm 0.1$ & $2.0 \pm 0.2$ & $340 \pm 30$ & $>>1000$ & $2.3 \pm 0.5$ & - & $240 \pm 30$ \\
\hline $\mathrm{dA}_{20}, \mathrm{H}_{2} \mathrm{O}, 266 \mathrm{~nm}$ & $1.0 \pm 0.1$ & $2.1 \pm 0.2$ & $190 \pm 20$ & $>>1000$ & $1.8 \pm 0.5$ & $44 \pm 10$ & $1200 \pm 200$ \\
\hline $\mathrm{dA}_{20}, \mathrm{H}_{2} \mathrm{O}, 285 \mathrm{~nm}$ & - & $4.0 \pm 0.4$ & $180 \pm 20$ & $>>1000$ & - & - & - \\
\hline $\mathrm{dA}_{1}, \mathrm{D}_{2} \mathrm{O}, 266 \mathrm{~nm}$ & $1.0 \pm 0.2$ & $2.5 \pm 0.5$ & - & - & - & - & - \\
\hline $\mathrm{dA}_{2}, \mathrm{D}_{2} \mathrm{O}, 266 \mathrm{~nm}$ & $1.0 \pm 0.1$ & $3.1 \pm 0.3$ & $730 \pm 70$ & $>>1000$ & $3.1 \pm 0.5$ & - & $270 \pm 30$ \\
\hline $\mathrm{dA}_{20}, \mathrm{D}_{2} \mathrm{O}, 266 \mathrm{~nm}$ & $1.0 \pm 0.1$ & $3.8 \pm 0.4$ & $330 \pm 30$ & $>>1000$ & $1.9 \pm 0.5$ & $35 \pm 10$ & $1300 \pm 200$ \\
\hline
\end{tabular}

Table 1. Time constants obtained from the multi-exponential fits performed for the TA and TAA data displayed in section S.3 and fig. S.4 in the SI. The time constants denoted $\tau_{i}$ are obtained from the transient absorption data by performing global fits of a sum of exponential decays, each convoluted with a Gaussian intrument response function. The time constants denoted $\rho_{i}$ refer to the anisotropy decays, where the GSB and ESA regions are fitted simultaneously with a sum of exponential decays.

obtained for $\mathrm{dA}_{20}(266 \mathrm{~nm})$ in $\mathrm{H}_{2} \mathrm{O}$ buffer solution. First of all we note that the associated DAS spectrum shows a uniform, but much lower contribution of the decay component in the ESA region, compared to the GSB region. This suggests that $\rho_{2}^{(20)}$ contributes to both TAA decays and is not simply caused by an overlap with the ESA band of the CT exciton. With global rotational diffusion of the strand accounted for by $\rho_{3}^{(20)}$, the orientation of a bleached transition dipole can only change if it moves from the position where it was initially photo-excited. We thus identify three possible processes to induce such a change: (1) a local rotational diffusion of the base stack, (2) exciton migration along the strand, and (3) a conformational change of the photoexcited base stack. Fluorescence anisotropy experiments have shown that the local conformational fluctuations of the central base within a tri-nucleotide in aqueous solution displays an anisotropy decay time constant of approximately $80 \mathrm{ps}^{54}$. The time constant measured here is notably shorter and is associated with a base stack, which thus suggests that a local fluctuation process is not a suitable explanation. In $\mathrm{dA}_{20}$, energy transfer of neutral excitons along the oligomer chain could in principle contribute to a decay of the GSB anisotropy. However, our previous discussion showed that internal conversion to the neutral exciton band minimum and charge separation of neutral excitons are completed in $<0.3 \mathrm{ps}$. These processes efficiently localize and trap the initially formed neutral excitons, which therefore excludes any intra-chain hopping on the picosecond timescale. The most probable origin of $\rho_{2}^{(20)}$ is thus an inter-base conformational change associated with the relaxation of the CT exciton. Note that the observed time scale is consistent with results from a similar study, where the structural relaxation of exciton states in $\pi$-stacked perylene-bis(dicarboximide) (PBI) H-aggregates was measured via TAA 
and fluorescence anisotropy 55 .

In order to determine the nature of this conformational change it is important to note how the motion of a photoexcited nucleotide impacts the anisotropy in the GSB and the ESA region. In the GSB region, the TAA decay tracks the motion of the bleached ${ }^{1} \pi \pi^{*}$ transition dipoles, which are initially parallel to the nucleobase planes. In comparison, the transition dipole of the CT exciton in the ESA region has an additional component perpendicular to the nucleobase planes. The DAS of $\rho_{2}$ shows that its kinetic contribution is significantly more pronounced in the TAA decay in the GSB rather than the ESA region. This suggests that the dominant conformational motion takes place in a plane parallel to the nucleobase planes, thus leading to a stronger TAA change in the GSB relative to the ESA region. Whilst we cannot exclude an additional reduction in inter-base distance as a possible structural relaxation pathway, we note that this would likely lead to a change of CT character and thus a pronounced change of the TAA in the ESA region. We therefore propose that $\rho_{2}$ is dominated by a reduction of the inter-base twist angle towards a parallel orientation, driving the relaxation of the CT exciton to its minimum energy conformation. A similar structural relaxation pathway was proposed by Son an co-workers for the exciton evolution in PBI aggregates $^{55}$. Furthermore, an inter-base rotation towards a parallel nucleotide alignment is qualitatively consistent with recent QM/MM simulations, which calculated minimum energy conformations of excited adenosine dimers incorporated into a solvated dsDNA geometry ${ }^{36}$.

In $\mathrm{D}_{2} \mathrm{O}$ buffer solution, the same qualitative behavior of the TAA decay is observed and comparable time constants are obtained from the global fits. First of all, this shows that the fitting procedure is robust across several data sets. Second, it shows that solvent deuteration has only a small impact on the rotational diffusion, as expected. Third, $\rho_{2}^{(20)}$ is unaffected by the solvent change within its error range, which is consistent with a change in inter-base conformation. In this view, however, it is somewhat surprising that $\rho_{2}$ is not observed in $\mathrm{dA}_{2}$. First of all, we found that it is possible to perform a global fit with three exponential decays on the TAA data in $\mathrm{H}_{2} \mathrm{O}$ and obtain a small amplitude contribution from an intermediate decay with $\rho_{2}^{(2)} \approx 50$ ps (fits not displayed). However, this could neither be achieved for repeated experiments, nor for the data set in $\mathrm{D}_{2} \mathrm{O}$, where the associated global fits did not converge to meaningful results. We thus speculate that whilst a structural relaxation of the CT exciton may also be present in $\mathrm{dA}_{2}$, it is likely to be less pronounced due to the weaker average electronic coupling of its 
nucleotides, compared to $\mathrm{dA}_{20}$.

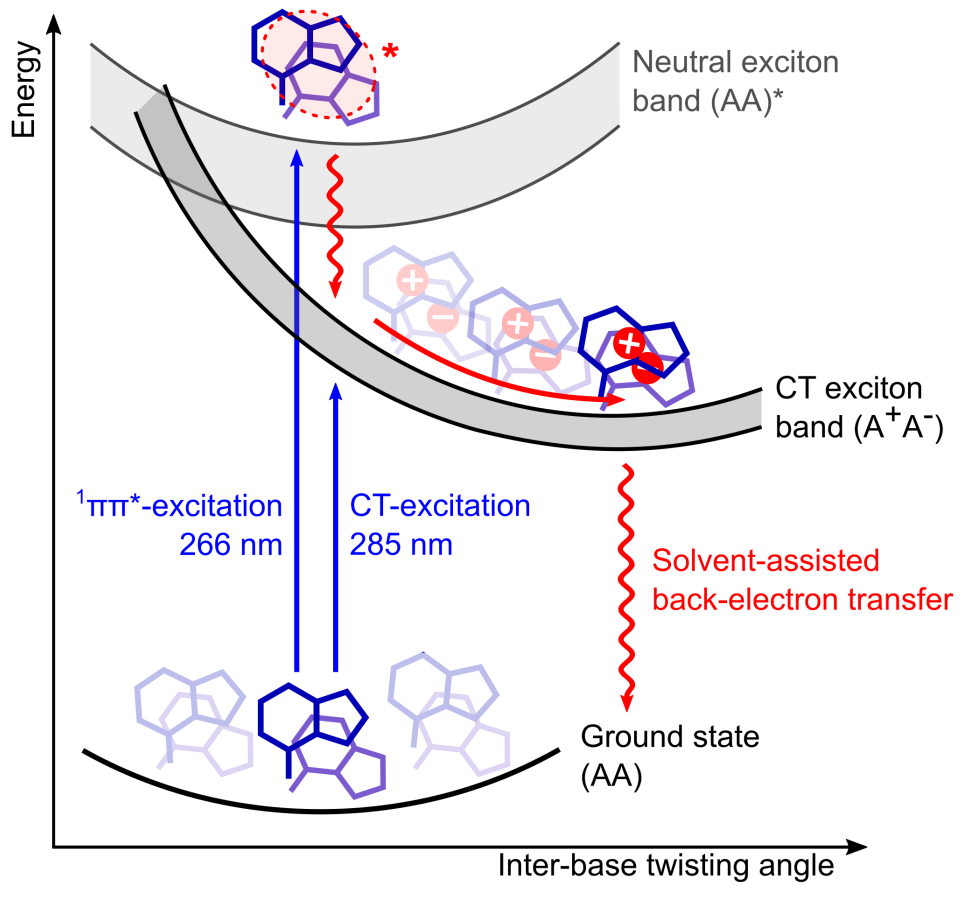

Figure 5. Schematic illustration of the photo-chemical relaxation mechanism in adenosine ssDNA $\left(\mathrm{dA}_{\mathrm{n}}\right)$. As inter-base electronic couplings are mostly restricted to nearest-neighbour stacked bases, sufficiently long $\mathrm{dA}_{\mathrm{n}}$ can be approximated as a chain of 2-base stacks. Photoexcitation of $\mathrm{dA}_{\mathrm{n}}$ thus selects a 2-base stack conformation from the fluctuating ground state conformational ensemble and predominantly forms neutral exciton states delocalized over two bases at the top of the exciton band. Most of these states undergo rapid intraband scattering and charge separation to form charge-transfer (CT) excitons in $<0.3 \mathrm{ps,}$ without any significant change in conformation. Ultrafast base rotations then drive the CT excitons to the minimum energy conformation of the CT exciton band in about $40 \mathrm{ps}$. From there a solvent-assisted back-electron transfer deactivates the exciton back to the ground state. Note that a small but significant fraction of the charge-separated states do not recombine and display a lifetime on the millisecond scale.

\section{Conclusion}

In summary, we are now able to present a complete reaction mechanism for the exciton dynamics in adenosine ssDNA, which is displayed in fig. 5. Upon photo-excitation, predominantly neutral excitons delocalized over two stacked bases are formed within the oligomer, with a stack conformation selected

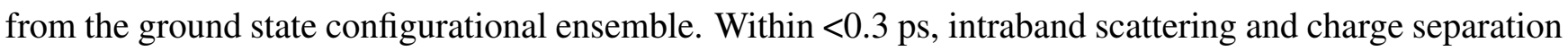
takes place without any significant conformational change, resulting in a CT exciton. As a consequence, the CT character is mostly determined by the inter-base distance upon photoexcitation. Afterwards, an ultrafast inter-base rotation drives the CT exciton to its minimum energy configuration in about 40 
ps, from where a solvent-assisted back-electron transfer leads to a charge recombination back to the ground. Importantly, this mechanism takes both ground and excited state structural properties of the oligomer into account and finally explains why short oligomers such as dimers display excitons with a lower CT character and slower recombination times: their average inter-base separation leads to a weaker electronic coupling and less efficient back-electron transfer. In addition, our results represent the first direct experimental observation of a structural mechanism in the ultrafast photochemistry of ssDNA, opening a novel perspective on its photodamage control processes. In a broader context, our results highlight the key role of ground and excited state structural features in the ultrafast photochemistry of aggregated organic molecular systems. This is expected to contribute to a more detailed understanding of the related energy and charge transfer processes in synthetic molecular aggregates.

\section{Methods}

\section{Broadband transient absorption and anisotropy in the deep-UV}

The laser setup for generating the deep-UV probe pulses has been described in detail elsewhere ${ }^{56,57}$. Briefly, a customized cryogenic Ti:Sapphire amplifier, with an exceptional shot-to-shot stability of $0.1 \%$ root-mean-square, pumps a commercial non-collinear optical parametric amplifier (NOPA) at $20 \mathrm{kHz}$.

The broadband visible femtosecond pulses are achromatically doubled in a thin BBO crystal ${ }^{58}$ covering 250-360 nm. The probe pulse is set to S-polarization with an achromatic half-wave plate before splitting it into a probe and a reference beam with a $1 \mathrm{~mm}$ thick reflective neutral density filter $(0.3 \mathrm{OD})$. The probe is focused into the sample with a $10 \mathrm{~cm}$ focal-length off-axis parabolic mirror, resulting in a focal spot diameter of approximately $30 \mu \mathrm{m}$ at full-width half maximum (FWHM). The probe polarization is cleaned with a high-quality Glan-Laser polarizer prior to detection with a fiber-coupled imaging spectrograph, which is equipped with two CMOS chips for recording the probe and reference simultaneously shot-to-shot. The sample is delivered via a wire-guided liquid jet with an approximate pathlength of $300 \mu \mathrm{m}$ (design adapted from ${ }^{59}$ ), in which a total volume of about $10 \mathrm{ml}$ is circulated in a closed loop. Solvent evaporation is accounted for by continuously replenishing it with a syringe pump. Two pump pulse configurations were employed: (1) a pump centered at $266 \mathrm{~nm}(\mathrm{FWHM} \approx 2 \mathrm{~nm})$, derived from the Ti:Sapphire amplifier via third harmonic generation, and (2) a pump centered at $285 \mathrm{~nm}(\mathrm{FWHM} \approx 2 \mathrm{~nm})$, obtained via narrowband 
second harmonic generation of the NOPA. The pump beam is focussed to a FWHM diameter of $80 \mu \mathrm{m}$ resulting in an estimated fluence $125 \mu \mathrm{J} \mathrm{cm}^{-2}$ on target. This was determined to be in the linear excitation regime via fluence-dependent transient absorption measurements. Due to a relatively large angle of about $10^{\circ}$ between the pump and probe beams and the use of a free sample jet, any pump scatter could be prevented from reaching the probe detector. The repetition rate of the pump is reduced to $10 \mathrm{kHz}$ via an optical chopper and its linear polarization is switched between $\mathrm{S}$ - and P-polarization via a motorized zero-order half-wave plate. At a given pump-probe delay t, 30,000 consecutive probe shots were acquired for each pump-polarization state, resulting in a TA spectrum for parallel pump and probe polarizations $\left(\mathrm{TA}^{\|}\right)$and a second for perpendicular polarizations $\left(\mathrm{TA}^{\perp}\right)$. The isotropic magic-angle TA and the TAA spectrum were then calculated as:

$$
\mathrm{TA}(\lambda, \mathrm{t})=\frac{1}{3}\left(\mathrm{TA}^{\|}(\lambda, \mathrm{t})+2 \mathrm{TA}^{\perp}(\lambda, \mathrm{t})\right), \quad \operatorname{TAA}^{\prime}(\lambda, \mathrm{t})=\frac{1}{3}\left(\mathrm{TA}^{\|}(\lambda, \mathrm{t})-\mathrm{TA}^{\perp}(\lambda, \mathrm{t})\right) \times(\mathrm{TA}(\lambda, \mathrm{t}))^{-1}
$$

The instrument response function (IRF) of the setup was determined by recording the two-photon absorption signal in the neat buffer solution. The data is displayed in fig. S.3 in the SI and displays the extracted FWHM of the Gaussian fits as a function of probe wavelength. For both pump configurations we find that the IRF is limited to about $0.3 \mathrm{ps}$ in the GSB region $(<280 \mathrm{~nm})$ and to $0.2 \mathrm{ps}$ in the ESA region $(>280 \mathrm{~nm})$. To quantify the TA and TAA kinetics, we performed a global analysis for each data set with the OPTIMUS software package ${ }^{60}$, simultaneously fitting multi-exponential functions convoluted with the Gaussian IRF over the probed spectral window.

\section{Sample preparation}

2'-Deoxyadenosine 5'-monophosphate $\left(\mathrm{dA}_{1}\right)$ was purchased from Sigma-Aldrich and $\mathrm{dA}_{2}$ and $\mathrm{dA}_{20}$ from biomers.net. The dimer and multimer consist of $\mathrm{dA}_{1}$ as building blocks with the phosphate group covalently linked to the deoxribose at the 3 ' carbon of the neighbouring $\mathrm{dA}_{1}$. From there a single strand is formed with a sugar phosphate backbone. The samples were used without any further purification and were dissolved in a phosphate-buffered saline purchased from Sigma-Aldrich, which was diluted with highly pure Milli-Q water or spectroscopy-grade deuterated water purchased from Sigma-Aldrich. This 
resulted in a buffer solution with a buffer concentration of $0.01 \mathrm{M}$, a sodium chloride concentration of $0.154 \mathrm{M}$ and a $\mathrm{pH}$ of 7.4. Additionally, samples were prepared in a methanol-water solution following the description $\mathrm{in}^{35}$, with $80 \mathrm{vol} \%$ of methanol and $20 \mathrm{vol} \%$ of Milli-Q water. The sample concentration was chosen to have an absorbance of $0.75 \mathrm{OD}$ at $260 \mathrm{~nm}$ in a $300 \mu \mathrm{m}$ path length. The sample integrity was characterized and confirmed before and after any experiment with a static UV-vis spectrometer (UV-3600, Shimadzu) and a static circular dichroism spectrometer (J-810, Jacso).

\section{Molecular dynamics simulations}

For the molecular dynamics (MD) simulations, initial single-stranded structures for both $d A_{2}$ and $d A_{20}$ were desined using the leap program of AMBERTOOLS $18^{61}$. Both molecules were solvated in a truncated octahedral box of explicit TIP3P ${ }^{62}$ water molecules, maintaining the water layer thickness greater than $11 \AA$ A surrounding the DNA molecule. First, $\mathrm{Na}^{+}$ions were added to neutralize the system, following the Joung and Cheatham ion model ${ }^{63}$. Second, then $\mathrm{Na}^{+}$and $\mathrm{Cl}^{-}$ion-pairs were added to reach an approximate overall salt concentration of $150 \mathrm{mM}$. The ions in the simulation box were placed randomly with the constraint that the ions should be at least $5 \AA$ away from the DNA molecule and at least $3.5 \AA$ from each other. The solvated systems thus obtained were first equilibrated to minimize the energy of the solvent and then subjected to a slow thermalization to reach room temperature followed by a further equilibration of 500 ps. Finally, 500 ns of production simulations were carried out in the NPT ensemble using a time-step of $2 \mathrm{fs}$ and storing the trajectory at a picosecond interval. All simulations were performed using the PARMBSC1 force field ${ }^{64}$ in AMBER18. The SHAKE algorithm ${ }^{65}$ was used to constrain the bond involving Hydrogen. For the long-range electrostatic interactions, the particle mesh Ewald method 66 was used with a $9 \AA$ real space cutoff. The short-range Lennard-Jones interactions were truncated at 9 A. Finally, AMBERTOOLS 18 was used to analyze the simulation results and to compute the electronic coupling element, $J=|\vec{J}|^{67}$. For two identical bases with subscripts 1 and 2 , it is defined as:

$$
J\left(R, \beta, \alpha_{1}, \alpha_{2}\right)=\frac{d^{2}}{R^{3}}\left[\cos (\beta)-3 \cos \left(\alpha_{1}\right) \cos \left(\alpha_{2}\right)\right]
$$

where $d=|\vec{d}|$ is the transition dipole moment of the base, $R=|\vec{R}|$ is the separation between two neighboring bases (defined as the vector between the centroids of the two bases), $\beta$ is the angle between the transition 
dipole moments of the two bases, and $\alpha_{i}$ is the angle between $\vec{d}_{i}$ and $\vec{R}$. The direction and the absolute value of $\vec{d}$ for Adenine (2.56 D) are taken from ${ }^{68}$.

\section{Acknowledgements}

The authors would like to thank Prof. John H. Maddocks from EPFL for his invaluable advice and support for the project. This work was supported by the Swiss NSF through the NCCR-MUST and MO in particular by a fellowship within the Postdoc-Program of the German Academic Exchange Service (DAAD). RS would like to acknowledge SCITAS for computational facilities and the Swiss National Science Foundation 200020182184.

\section{References}

1. Brixner, T., Hildner, R., Köhler, J., Lambert, C. \& Würthner, F. Exciton Transport in Molecular Aggregates - From Natural Antennas to Synthetic Chromophore Systems. Adv. Energy Mater. 7, n/a-n/a, DOI: 10.1002/aenm.201700236 (2017).

2. Kasha, M., Rawls, H. R. \& Ashraf El-Bayoumi, M. The exciton model in molecular spectroscopy. Pure Appl. Chem. 11, DOI: 10.1351/pac196511030371 (1965).

3. Schreier, W. J., Gilch, P. \& Zinth, W. Early Events of DNA Photodamage. Annu. Rev. Phys. Chem. 66, 497-519, DOI: 10.1146/annurev-physchem-040214-121821 (2015).

4. Chen, J., Zhang, Y. \& Kohler, B. Excited States in DNA Strands Investigated by Ultrafast Laser Spectroscopy. In Barbatti, M., Borin, A. C. \& Ullrich, S. (eds.) Photoinduced Phenomena in Nucleic Acids II: DNA Fragments and Phenomenological Aspects, 39-87, DOI: 10.1007/128_2014_570 (Springer International Publishing, Cham, 2015).

5. Markovitsi, D. UV-induced DNA Damage: The Role of Electronic Excited States. Photochem. Photobiol. 92, 45-51, DOI: 10.1111/php.12533 (2016).

6. Plasser, F. \& Lischka, H. Analysis of Excitonic and Charge Transfer Interactions from Quantum Chemical Calculations. J. Chem. Theory Comput. 8, 2777-2789, DOI: 10.1021/ct300307c (2012).

7. Blancafort, L. \& Voityuk, A. A. Exciton delocalization, charge transfer, and electronic coupling for singlet excitation energy transfer between stacked nucleobases in DNA: An MS-CASPT2 study. The J. Chem. Phys. 140, 095102, DOI: 10.1063/1.4867118 (2014).

8. Improta, R., Santoro, F. \& Blancafort, L. Quantum Mechanical Studies on the Photophysics and the Photochemistry of Nucleic Acids and Nucleobases. Chem. Rev. 116, 3540-3593, DOI: 10.1021/acs. chemrev.5b00444 (2016).

9. Olsthoorn, C. S. M., Bostelaar, L. J., Rooij, J. F. M. D., Boom, J. H. V. \& Altona, C. Circular Dichroism Study of Stacking Properties of Oligodeoxyadenylates and Polydeoxyadenylate. Eur. J. Biochem. 115, 309-321, DOI: 10.1111/j.1432-1033.1981.tb05240.x (1981). 
10. Barbatti, M. \& Ullrich, S. Ionization potentials of adenine along the internal conversion pathways. Phys. Chem. Chem. Phys. 13, 15492-15500, DOI: 10.1039/C1CP21350D (2011).

11. Gustavsson, T. et al. A joint experimental/theoretical study of the ultrafast excited state deactivation of deoxyadenosine and 9-methyladenine in water and acetonitrile. Photochem. \& Photobiol. Sci. 12, 1375-1386, DOI: 10.1039/C3PP50060H (2013).

12. Pecourt, J.-M. L., Peon, J. \& Kohler, B. Ultrafast Internal Conversion of Electronically Excited RNA and DNA Nucleosides in Water. J. Am. Chem. Soc. 122, 9348-9349, DOI: 10.1021/ja0021520 (2000).

13. Spano, F. C. Excitons in conjugated oligomer aggregates, films, and crystals. Annu. Rev. Phys. Chem. 57, 217-243, DOI: 10.1146/annurev.physchem.57.032905.104557 (2006).

14. Hu, L. et al. Are Adenine Strands Helical H-Aggregates? The J. Phys. Chem. B 111, 11812-11816, DOI: 10.1021/jp070403m (2007).

15. Tinoco, I. Hypochromism in Polynucleotides ${ }^{1}$. J. Am. Chem. Soc. 82, 4785-4790, DOI: 10.1021/ ja01503a007 (1960).

16. Eisinger, J., Guéron, M., Shulman, R. G. \& Yamane, T. Excimer fluorescence of dinucleotides, polynucleotides, and DNA. Proc. Natl. Acad. Sci. United States Am. 55, 1015-1020 (1966).

17. Tonzani, S. \& Schatz, G. C. Electronic Excitations and Spectra in Single-Stranded DNA. J. Am. Chem. Soc. 130, 7607-7612, DOI: 10.1021/ja7103894 (2008).

18. Ai, Y.-J., Cui, G.-L., Fang, Q., Fang, W.-H. \& Luo, Y. Exploring concerted effects of base pairing and stacking on the excited-state nature of DNA oligonucleotides by DFT and TD-DFT studies. Int. J. Quantum Chem. 111, 2366-2377, DOI: 10.1002/qua.22524 (2011).

19. Improta, R. \& Barone, V. Interplay between "Neutral" and "Charge-Transfer" Excimers Rules the Excited State Decay in Adenine-Rich Polynucleotides. Angewandte Chemie Int. Ed. 50, 12016-12019, DOI: 10.1002/anie.201104382 (2011).

20. Nogueira, J. J., Plasser, F. \& González, L. Electronic delocalization, charge transfer and hypochromism in the UV absorption spectrum of polyadenine unravelled by multiscale computations and quantitative wavefunction analysis. Chem. Sci. 8, 5682-5691, DOI: 10.1039/C7SC01600J (2017).

21. Kadhane, U., Holm, A. I. S., Hoffmann, S. V. \& Nielsen, S. B. Strong coupling between adenine nucleobases in DNA single strands revealed by circular dichroism using synchrotron radiation. Phys. Rev. E 77, 021901, DOI: 10.1103/PhysRevE.77.021901 (2008).

22. Banyasz, A. et al. Multi-Pathway Excited State Relaxation of Adenine Oligomers in Aqueous Solution: A Joint Theoretical and Experimental Study. Chem. A Eur. J. 19, 3762-3774, DOI: 10.1002/chem.201202741 (2013).

23. Avila Ferrer, F. J., Improta, R., Santoro, F. \& Barone, V. Computing the inhomogeneous broadening of electronic transitions in solution: a first-principle quantum mechanical approach. Phys. Chem. Chem. Phys. 13, 17007-17012, DOI: 10.1039/C1CP22115A (2011).

24. Yin, H., Ma, Y., Mu, J., Liu, C. \& Rohlfing, M. Charge-Transfer Excited States in Aqueous DNA: Insights from Many-Body Green's Function Theory. Phys. Rev. Lett. 112, 228301, DOI: 10.1103/PhysRevLett.112.228301 (2014). Publisher: American Physical Society.

25. Kwok, W.-M., Ma, C. \& Phillips, D. L. Femtosecond Time- and Wavelength-Resolved Fluorescence and Absorption Spectroscopic Study of the Excited States of Adenosine and an Adenine Oligomer. $J$. Am. Chem. Soc. 128, 11894-11905, DOI: 10.1021/ja0622002 (2006). 
26. Buchvarov, I., Wang, Q., Raytchev, M., Trifonov, A. \& Fiebig, T. Electronic energy delocalization and dissipation in single-and double-stranded DNA. Proc. Natl. Acad. Sci. 104, 4794-4797 (2007).

27. Takaya, T., Su, C., de La Harpe, K., Crespo-Hernández, C. E. \& Kohler, B. UV excitation of single DNA and RNA strands produces high yields of exciplex states between two stacked bases. Proc. Natl. Acad. Sci. 105, 10285-10290 (2008).

28. Su, C., Middleton, C. T. \& Kohler, B. Base-Stacking Disorder and Excited-State Dynamics in Single-Stranded Adenine Homo-oligonucleotides. The J. Phys. Chem. B 116, 10266-10274, DOI: 10.1021/jp305350t (2012).

29. Borrego-Varillas, R., Cerullo, G. \& Markovitsi, D. Exciton Trapping Dynamics in DNA Multimers. The J. Phys. Chem. Lett. 10, 1639-1643, DOI: 10.1021/acs.jpclett.9b00450 (2019).

30. Bucher, D. B., Pilles, B. M., Carell, T. \& Zinth, W. Charge separation and charge delocalization identified in long-living states of photoexcited DNA. Proc. Natl. Acad. Sci. 111, 4369-4374, DOI: 10.1073/pnas.1323700111 (2014).

31. Zhang, Y. et al. Efficient UV-induced charge separation and recombination in an 8-oxoguaninecontaining dinucleotide. Proc. Natl. Acad. Sci. 111, 11612-11617, DOI: 10.1073/pnas.1404411111 (2014).

32. Banyasz, A. et al. UV-Induced Adenine Radicals Induced in DNA A-Tracts: Spectral and Dynamical Characterization. The J. Phys. Chem. Lett. 7, 3949-3953, DOI: 10.1021/acs.jpclett.6b01831 (2016).

33. Banyasz, A., Ketola, T., Martínez-Fernández, L., Improta, R. \& Markovitsi, D. Adenine radicals generated in alternating AT duplexes by direct absorption of low-energy UV radiation. Faraday Discuss. 207, 181-197, DOI: 10.1039/C7FD00179G (2018).

34. Conti, I. et al. Excited state evolution of DNA stacked adenines resolved at the CASPT2//CASSCF/Amber level: from the bright to the excimer state and back. Phys. Chem. Chem. Phys. 17, 7291-7302, DOI: 10.1039/C4CP05546B (2015). Publisher: Royal Society of Chemistry.

35. Chen, J. \& Kohler, B. Base Stacking in Adenosine Dimers Revealed by Femtosecond Transient Absorption Spectroscopy. J. Am. Chem. Soc. 136, 6362-6372, DOI: 10.1021/ja501342b (2014).

36. Spata, V. A. \& Matsika, S. Photophysical deactivation pathways in adenine oligonucleotides. Phys. Chem. Chem. Phys. 17, 31073-31083, DOI: 10.1039/C5CP04254B (2015).

37. Plasser, F. \& Lischka, H. Electronic excitation and structural relaxation of the adenine dinucleotide in gas phase and solution. Photochem. \& Photobiol. Sci. 12, 1440-1452, DOI: 10.1039/C3PP50032B (2013).

38. Hub, J. S. Interpreting solution X-ray scattering data using molecular simulations. Curr. Opin. Struct. Biol. 49, 18-26, DOI: 10.1016/j.sbi.2017.11.002 (2018).

39. Sugiki, T., Kobayashi, N. \& Fujiwara, T. Modern Technologies of Solution Nuclear Magnetic Resonance Spectroscopy for Three-dimensional Structure Determination of Proteins Open Avenues for Life Scientists. Comput. Struct. Biotechnol. J. 15, 328-339, DOI: 10.1016/j.csbj.2017.04.001 (2017).

40. Segarra-Martí, J. et al. Two-dimensional electronic spectroscopy as a tool for tracking molecular conformations in DNA/RNA aggregates. Faraday Discuss. 207, 233-250, DOI: 10.1039/C7FD00201G (2018). 
41. Stange, U. C. \& Temps, F. Ultrafast electronic deactivation of UV-excited adenine and its riboand deoxyribonucleosides and -nucleotides: A comparative study. Chem. Phys. 515, 441-451, DOI: 10.1016/j.chemphys.2018.08.031 (2018).

42. Ruckebusch, C., Sliwa, M., Pernot, P., de Juan, A. \& Tauler, R. Comprehensive data analysis of femtosecond transient absorption spectra: A review. J. Photochem. Photobiol. C: Photochem. Rev. 13, 1-27, DOI: 10.1016/j.jphotochemrev.2011.10.002 (2012).

43. Crespo-Hernández, C. E., Cohen, B. \& Kohler, B. Base stacking controls excited-state dynamics in A.T DNA. Nature 436, 1141-1144, DOI: 10.1038/nature03933 (2005).

44. de La Harpe, K., Crespo-Hernández, C. E. \& Kohler, B. Deuterium Isotope Effect on ExcitedState Dynamics in an Alternating GC Oligonucleotide. J. Am. Chem. Soc. 131, 17557-17559, DOI: 10.1021/ja9076364 (2009). Publisher: American Chemical Society.

45. Bucher, D. B., Schlueter, A., Carell, T. \& Zinth, W. Watson-Crick Base Pairing Controls Excited-State Decay in Natural DNA. Angewandte Chemie Int. Ed. 53, 11366-11369, DOI: 10.1002/anie.201406286 (2014).

46. Zhang, Y., de La Harpe, K., Beckstead, A. A., Improta, R. \& Kohler, B. UV-Induced Proton Transfer between DNA Strands. J. Am. Chem. Soc. 137, 7059-7062, DOI: 10.1021/jacs.5b03914 (2015).

47. Gould, I. R. \& Farid, S. Specific deuterium isotope effects on the rates of electron transfer within geminate radical-ion pairs. J. Am. Chem. Soc. 110, 7883-7885, DOI: 10.1021/ja00231a053 (1988).

48. Krishtalik, L. I. The mechanism of the proton transfer: an outline. Biochimica et Biophys. Acta (BBA) - Bioenerg. 1458, 6-27, DOI: 10.1016/S0005-2728(00)00057-8 (2000).

49. Smith, V. R., Samoylova, E., Ritze, H.-H., Radloff, W. \& Schultz, T. Excimer states in microhydrated adenine clusters. Phys. Chem. Chem. Phys. 12, 9632-9636, DOI: 10.1039/C003967E (2010). Publisher: Royal Society of Chemistry.

50. Kettani, A., Guéron, M. \& Leroy, J.-L. Amino Proton Exchange Processes in Mononucleosides. J. Am. Chem. Soc. 119, 1108-1115, DOI: 10.1021/ja962022k (1997). Publisher: American Chemical Society.

51. Beecroft, R. A. et al. A solvent isotope effect upon exciplex photochemistry. Chem. Phys. Lett. 93, 468-473, DOI: 10.1016/0009-2614(82)83222-3 (1982).

52. Mataga, N., Chosrowjan, H. \& Taniguchi, S. Ultrafast charge transfer in excited electronic states and investigations into fundamental problems of exciplex chemistry: Our early studies and recent developments. J. Photochem. Photobiol. C: Photochem. Rev. 6, 37-79, DOI: 10.1016/j.jphotochemrev. 2005.02.003 (2005).

53. Takaya, T., Hamaguchi, H.-o. \& Iwata, K. Femtosecond time-resolved absorption anisotropy spectroscopy on 9,9'-bianthryl: Detection of partial intramolecular charge transfer in polar and nonpolar solvents. The J. Chem. Phys. 130, 014501, DOI: 10.1063/1.3043368 (2009).

54. Jean, J. M. \& Krueger, B. P. Structural Fluctuations and Excitation Transfer between Adenine and 2-Aminopurine in Single-Stranded Deoxytrinucleotides. The J. Phys. Chem. B 110, 2899-2909, DOI: 10.1021/jp054755+ (2006). Publisher: American Chemical Society.

55. Son, M., Park, K. H., Shao, C., Würthner, F. \& Kim, D. Spectroscopic Demonstration of Exciton Dynamics and Excimer Formation in a Sterically Controlled Perylene Bisimide Dimer Aggregate. The J. Phys. Chem. Lett. 5, 3601-3607, DOI: 10.1021/jz501953a (2014). 
56. Auböck, G., Consani, C., Mourik, F. v. \& Chergui, M. Ultrabroadband femtosecond two-dimensional ultraviolet transient absorption. Opt. Lett. 37, 2337-2339, DOI: 10.1364/OL.37.002337 (2012).

57. Auböck, G. et al. Femtosecond pump/supercontinuum-probe setup with $20 \mathrm{kHz}$ repetition rate. Rev. Sci. Instruments 83, 093105, DOI: 10.1063/1.4750978 (2012).

58. Baum, P., Lochbrunner, S. \& Riedle, E. Tunable sub-10-fs ultraviolet pulses generated by achromatic frequency doubling. Opt. Lett. 29, 1686-1688, DOI: 10.1364/OL.29.001686 (2004).

59. Picchiotti, A., Prokhorenko, V. I. \& Miller, R. J. D. A closed-loop pump-driven wire-guided flow jet for ultrafast spectroscopy of liquid samples. Rev. Sci. Instruments 86, 093105, DOI: 10.1063/1.4929860 (2015).

60. Slavov, C., Hartmann, H. \& Wachtveitl, J. Implementation and Evaluation of Data Analysis Strategies for Time-Resolved Optical Spectroscopy. Anal. Chem. 87, 2328-2336, DOI: 10.1021/ac504348h (2015).

61. Case, D. et al. Amber 2018; 2018. Univ. California, San Francisco .

62. Jorgensen, W. L., Chandrasekhar, J., Madura, J. D., Impey, R. W. \& Klein, M. L. Comparison of simple potential functions for simulating liquid water. The J. chemical physics 79, 926-935 (1983).

63. Joung, I. S. \& Cheatham III, T. E. Determination of alkali and halide monovalent ion parameters for use in explicitly solvated biomolecular simulations. The journal physical chemistry B 112, 9020-9041 (2008).

64. Ivani, I. et al. Parmbsc1: a refined force field for DNA simulations. Nat. methods 13, 55 (2016).

65. Ryckaert, J.-P., Ciccotti, G. \& Berendsen, H. J. Numerical integration of the cartesian equations of motion of a system with constraints: molecular dynamics of n-alkanes. J. computational physics $\mathbf{2 3}$, 327-341 (1977).

66. Darden, T., York, D. \& Pedersen, L. Particle mesh Ewald: An N.log(N) method for Ewald sums in large systems. The J. chemical physics 98, 10089-10092 (1993).

67. Seibt, J. et al. On the geometry dependence of molecular dimer spectra with an application to aggregates of perylene bisimide. Chem. physics 328, 354-362 (2006).

68. Svozil, D., Jungwirth, P. \& Havlas, Z. Electron binding to nucleic acid bases. experimental and theoretical studies. a review. Collect. Czechoslov. chemical communications 69, 1395-1428 (2004). 


\section{Supporting information}

\section{Contents}

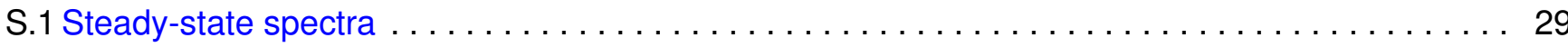

S.2 Two-photon absorption in buffer solution $\ldots \ldots \ldots \ldots \ldots \ldots \ldots \ldots \ldots \ldots \ldots \ldots \ldots \ldots \ldots$

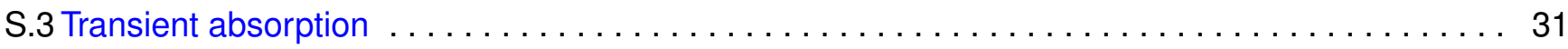

S.4 Transient absorption anisotropy $\ldots \ldots \ldots \ldots \ldots \ldots \ldots \ldots \ldots \ldots \ldots \ldots \ldots \ldots \ldots \ldots \ldots$

This supplementary information provides technical details concerning the performed experiments and their analysis. In the first section, the steady-state absorption and circular dichroism spectra of the investigated samples are displayed. In the second section, the two-photon absorption signal in a neat buffer solution is presented in order to assess the time-resolution of the presented experiments. In the third and fourth sections, the data analysis and fitting procedures for the transient absorption (TA), respectively anisotropy (TAA) measurements are discussed and evaluated. 


\section{S.1 Steady-state spectra}

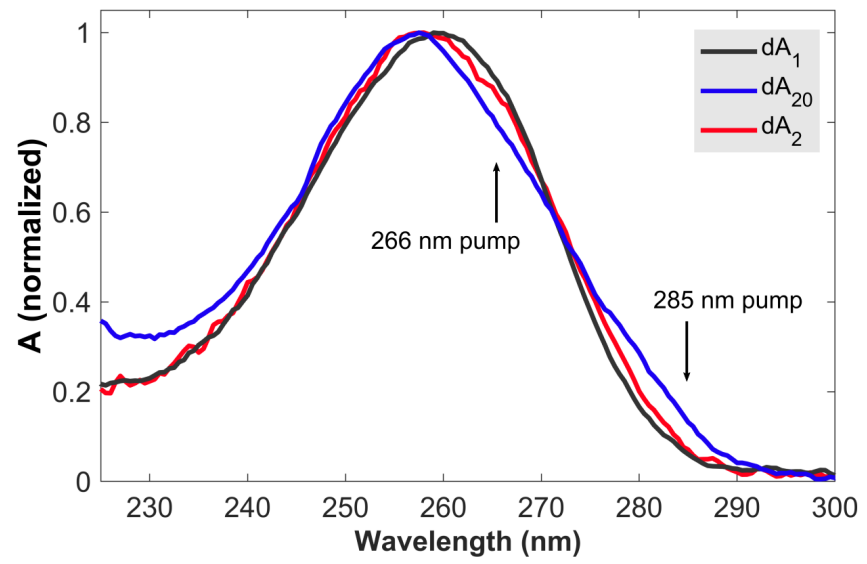

Figure S.1. Steady-state absorption spectra of $\mathrm{dA}_{1}, \mathrm{dA}_{20}$, and $\mathrm{dA}_{2}$ in aqueous phosphate buffer solution, each normalized to its absorption maximum. The spectral differences illustrate the impact of dipole-dipole and charge transfer interactions between the adenosine bases.
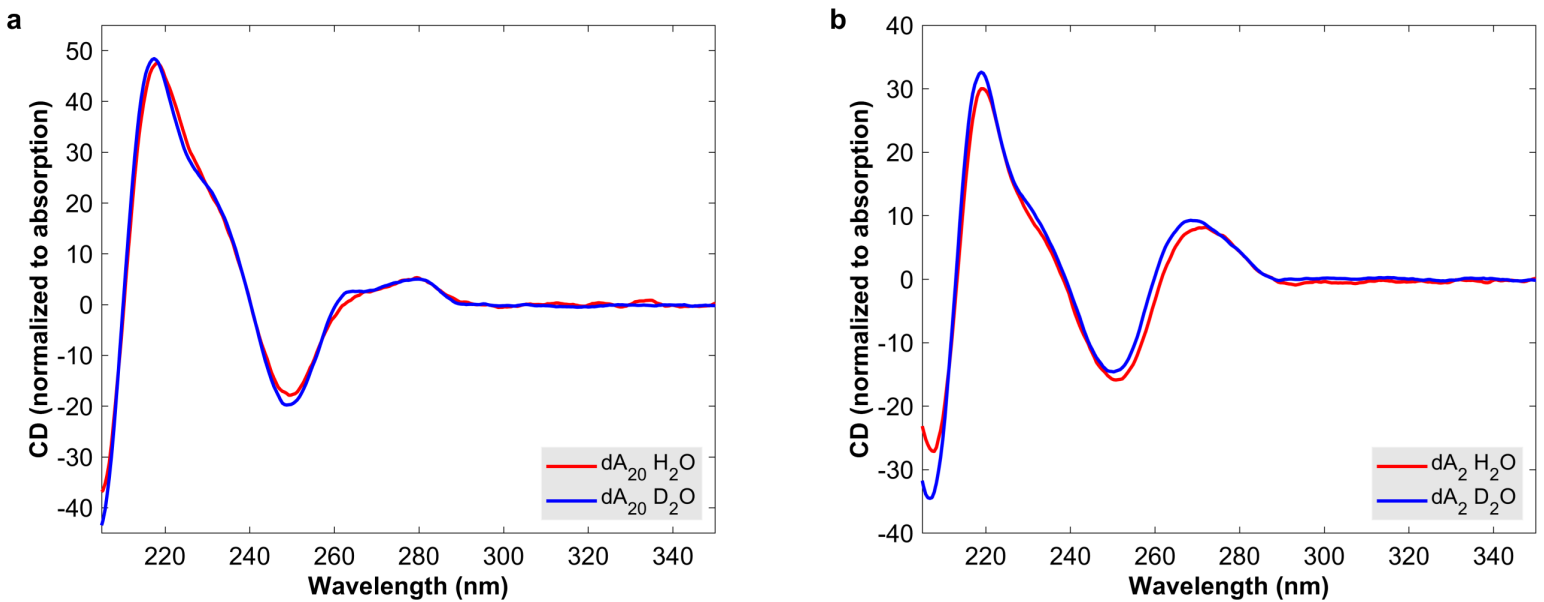

Figure S.2. a) Circular dichroism (CD) spectra of $\mathrm{dA}_{20}$ in $\mathrm{H}_{2} \mathrm{O}$ and $\mathrm{D}_{2} \mathrm{O}$ buffer solution. To account for possible solvent impacts on the absorption coefficient, the $\mathrm{CD}$ spectra are normalized to the absorption maximum near $260 \mathrm{~nm}$ for each sample. b) $\mathrm{CD}$ spectra of $\mathrm{dA}_{20}$ in $\mathrm{H}_{2} \mathrm{O}$ and $\mathrm{D}_{2} \mathrm{O}$ buffer solution with the same normalization procedure.

\section{S.2 Two-photon absorption in buffer solution}

In order to determine the instrument response function (IRF) of the TA and TAA measurements presented in the main text, the nonlinear response of the neat solvent at pump-probe overlap was recorded. Here, the employed pump fluence of appoximately $300 \mu \mathrm{J} \mathrm{cm}^{-2}$ was more than doubled compared to the presented sample measurements, in order to achieve a good signal-to-noise. The TA is displayed as a 
a
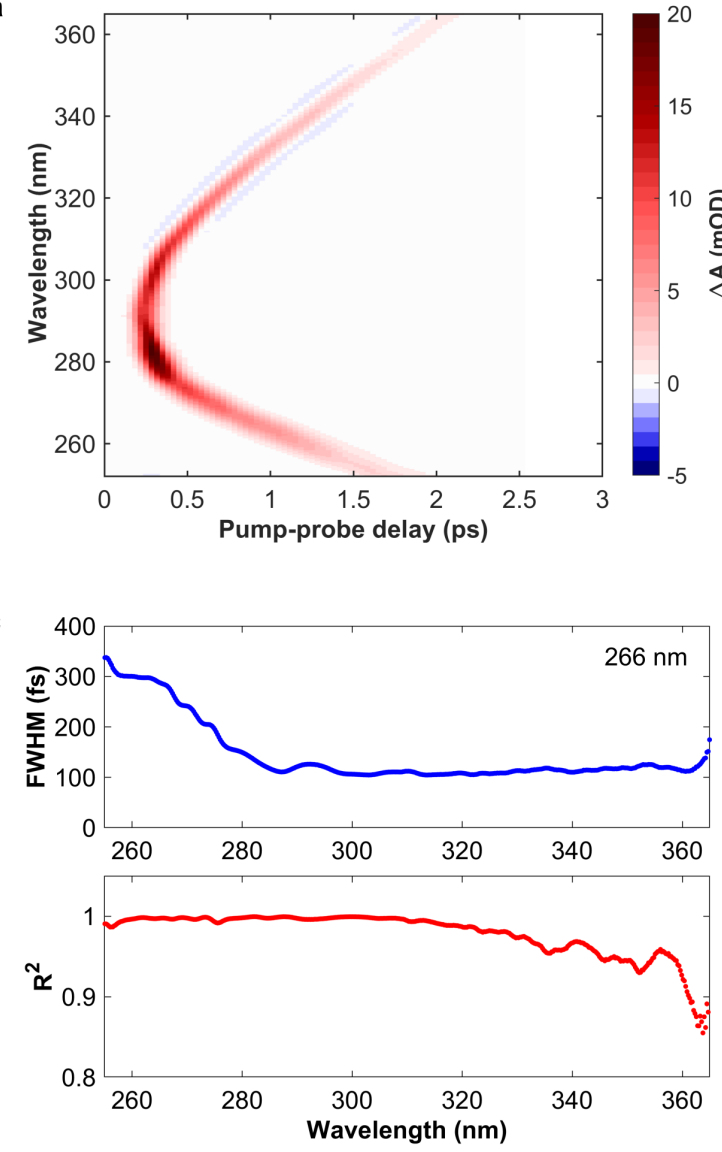

b

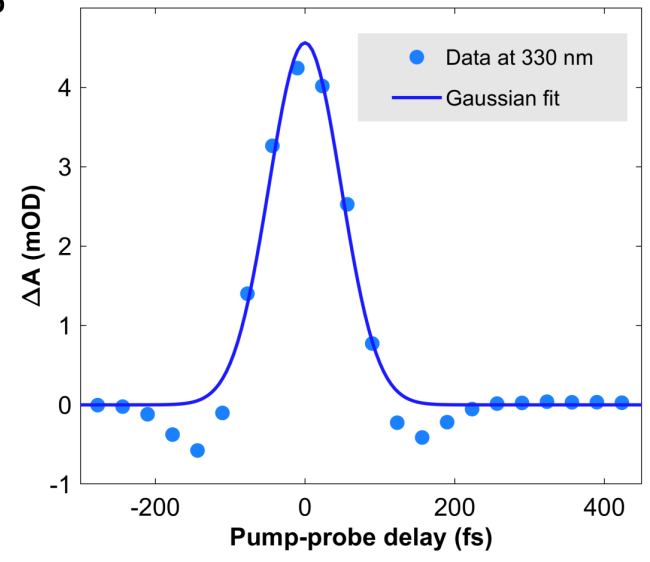

d
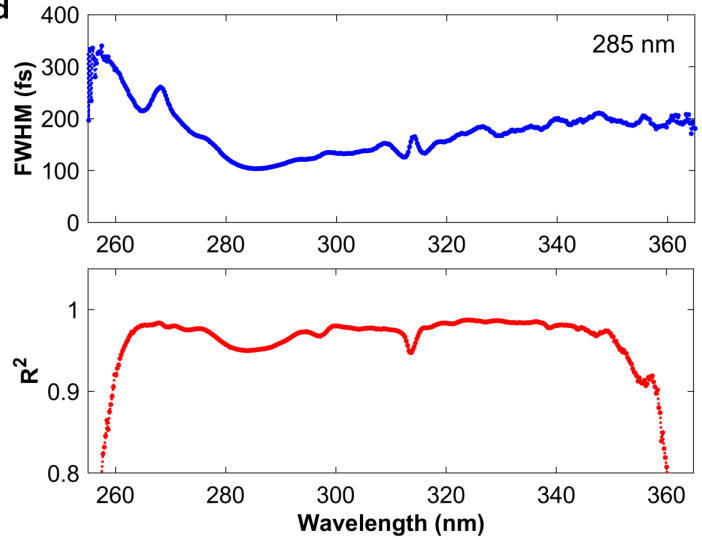

Figure S.3. a) displays the transient absorption time-wavelength map of a neat $\mathrm{H}_{2} \mathrm{O}$ buffer solution excited with a pump pulse centred at $266 \mathrm{~nm}$ and with an estimated fluence of $300 \mu \mathrm{J} \mathrm{cm}{ }^{-2}$. The nonlinear solvent response is dominated by two-photon absorption during the temporal overlap of the pump and probe pulses, which can be fitted with a Gaussian function. b) Kinetic trace obtained from (a) at a probe wavelength of $330 \mathrm{~nm}$ and the corresponding Gaussian fit. c) Full-width at half maximum (FWHM) of the performed Gaussian fits as a function of probe wavelength and the associated $\mathrm{R}^{2}$ value obtained for each fit for a $266 \mathrm{~nm}$ pump pulse. The obtained FWHM correspond to the instrument response function (IRF) quoted in the main text. d) FWHM and fit quality obtained for a $285 \mathrm{~nm}$ pump pulse.

time-wavelength map in fig. S.3a, which shows a parabolic pump-probe delay dependence of the temporal overlap of the spectral components of the probe pulse. This effect is caused by the probe pulse's group velocity dispersion (GVD), which is routinely corrected in the post-processing of transient absorption data. Fig. S.3a shows an exemplary time trace taken from the time-wavelength map at $330 \mathrm{~nm}$. It shows that the solvent response is dominated by a strong positive signal mostly caused by two-photon absorption from the pump and probe pulses. There is an additional minor negative signal contribution that is caused by cross-phase modulation. The IRF is estimated as the full-width at half maximum (FWHM) from a Gaussian function fitted to the individual time traces at each probe wavelength. The resulting FWHM 
values as a function of the probe wavelength are displayed in fig. S.3c,d, for pump pulses centered at 266 $\mathrm{nm}$ and $285 \mathrm{~nm}$ respectively, along with the associated $\mathrm{R}^{2}$ value. Whilst the IRF is $<150 \mathrm{fs}$ in the spectral region $>280 \mathrm{~nm}$, it increases to about 350 fs for shorter wavelengths. This is most likely caused by the increase in dispersion for shorter wavelengths in commonly employed optical materials. In this context, it should be noted that fig. S.3b illustrates that the shortest time-delay that can be probed without any signal from the solvent is approximately $250 \mathrm{fs}$ from the centre of two-photon absorption signal.

\section{S.3 Transient absorption}

Before any global fits were performed, the time-wavelength TA maps were GVD corrected, making use of the prominent two-photon absorption signal at pump-probe overlap. As the main concern of the analysis is to extract the dynamics of the charge-transfer (CT) exciton on the picosecond time scale, data points below 0.4 picoseconds were disregarded, thereby excluding contributions from two-photon absorption and cross-phase modulation near the pump-probe overlap. As a consequence, kinetics on the sub-picosecond time scale were not resolved in the quantitative analysis. The TA data was fitted with a sequence of exponential decays convoluted with a Gaussian IRF, using the OPTIMUS software package ${ }^{60}$. In all cases, the minimum number of exponential decays to achieve a suitable fit was applied, as explained in the main text. Fig. S.4 displays the time-wavelength TA maps, the obtained decay associated spectra (DAS) for $\mathrm{dA}_{1}(266 \mathrm{~nm}), \mathrm{dA}_{2}(266 \mathrm{~nm})$, and $\mathrm{dA}_{20}(266 \mathrm{~nm})$ in $\mathrm{H}_{2} \mathrm{O}$ buffer solution. To evaluate the quality of the global fits, fig. S.5 displays the associated time-wavelength maps of the obtained residuals and time traces of the TA amplitude, which compare the fit to the data points at selected probe wavelengths. Fig. S.6 and S.7 then show the same information for these samples in $\mathrm{D}_{2} \mathrm{O}$ buffer solution, whereas fig. S.9 displays the results for $\mathrm{dA}_{20}(285 \mathrm{~nm})$ in $\mathrm{H}_{2} \mathrm{O}$ buffer solution. Note that $\mathrm{dA}_{20}(285 \mathrm{~nm})$ displays a particularly low signal amplitude due to the low absorption coefficient at the excitation wavelength of $285 \mathrm{~nm}$. To achieve a good signal-to-noise, the number of sampled pump-probe delays was reduced to accumulate a higher number of pump-probe scans.

In the case of $\mathrm{dA}_{1}(266 \mathrm{~nm})$ in $\mathrm{H}_{2} \mathrm{O}$, two exponential decays were required to achieve an appropriate fit quality, accounting for the observed spectral shifts. The corresponding residuals are highest in the spectral range which is dominated by vibrational cooling dynamics and at most around $2 \%$. This is 
consistent with the well-known observation that spectral shifts cannot be modeled quantitatively by global multi-exponential fits. However, for the presented analysis the achieved fit quality is sufficient to describe the kinetics of the vibrational cooling dynamics without distorting the TA kinetics on longer time scales. In particular, the exciton relaxation dynamics in $\mathrm{dA}_{2}(266 \mathrm{~nm})$ and $\mathrm{dA}_{20}(266 \mathrm{~nm})$ take place on time scales that are two orders of magnitude slower. Following previously published results on the relaxation kinetics in $\mathrm{dA}_{\mathrm{n}}{ }^{27,28}$, two decay components were added to the exponential decay sequence in $\mathrm{A}_{2}(266$ $\mathrm{nm})$ and $\mathrm{dA}_{20}(266 \mathrm{~nm})$ : a component on the $100 \mathrm{ps}$ scale describing the relaxation of the CT exciton and a component with a time constant $\gg 1 \mathrm{~ns}$ accounting for long-lived radical species ${ }^{32}$. We achieve a very good fit quality with low residuals, which are $<1 \%$ across the probed spectral range for pump-probe delays $>10$ ps. Here, the lowest residuals are achieved for $\mathrm{dA}_{20}$, because it displays negligible vibrational cooling dynamics as explained in the main text. Due to the high quality of the global fits, we assign a $10 \%$ error to each of the obtained decay constants. To avoid any over-fitting of the data and corroborate the choice of four exponential decay components, we took $\mathrm{dA}_{2}$ as an exemplary case and removed exponential components from the global fit in a step-wise fashion. Fig. S.8 then compares the residuals and time traces for global fits with four, three and two components. Removing the longest decay component $\tau_{4}($ fig. S.8b,e) results in significantly larger residuals $>100 \mathrm{ps,} \mathrm{whereas} \mathrm{the} \mathrm{additional} \mathrm{removal} \mathrm{of} \mathrm{the} \mathrm{sub-picosecond}$ component $\tau_{1}$ leads to a global mismatch between the data and the fits (fig. S.8c,d). This confirms that four decay components are required to achieve an adequate fit of the oligomer data sets.

For the samples in $\mathrm{D}_{2} \mathrm{O}$ buffer solution comparable fit qualities are obtained, as is visualized by the low residuals. However, the solvent deuteration has a significant impact on the observed TA kinetics. Whilst slower vibrational cooling dynamics are already observed in $\mathrm{dA}_{1}, \mathrm{~A}_{2}$ and $\mathrm{dA}_{20}$ also display a much longer CT exciton relaxation time. Finally, the fits obtained for $\mathrm{dA}_{20}(285 \mathrm{~nm})$ in $\mathrm{H}_{2} \mathrm{O}$ buffer solution show a similar quality as the previously discussed samples. 

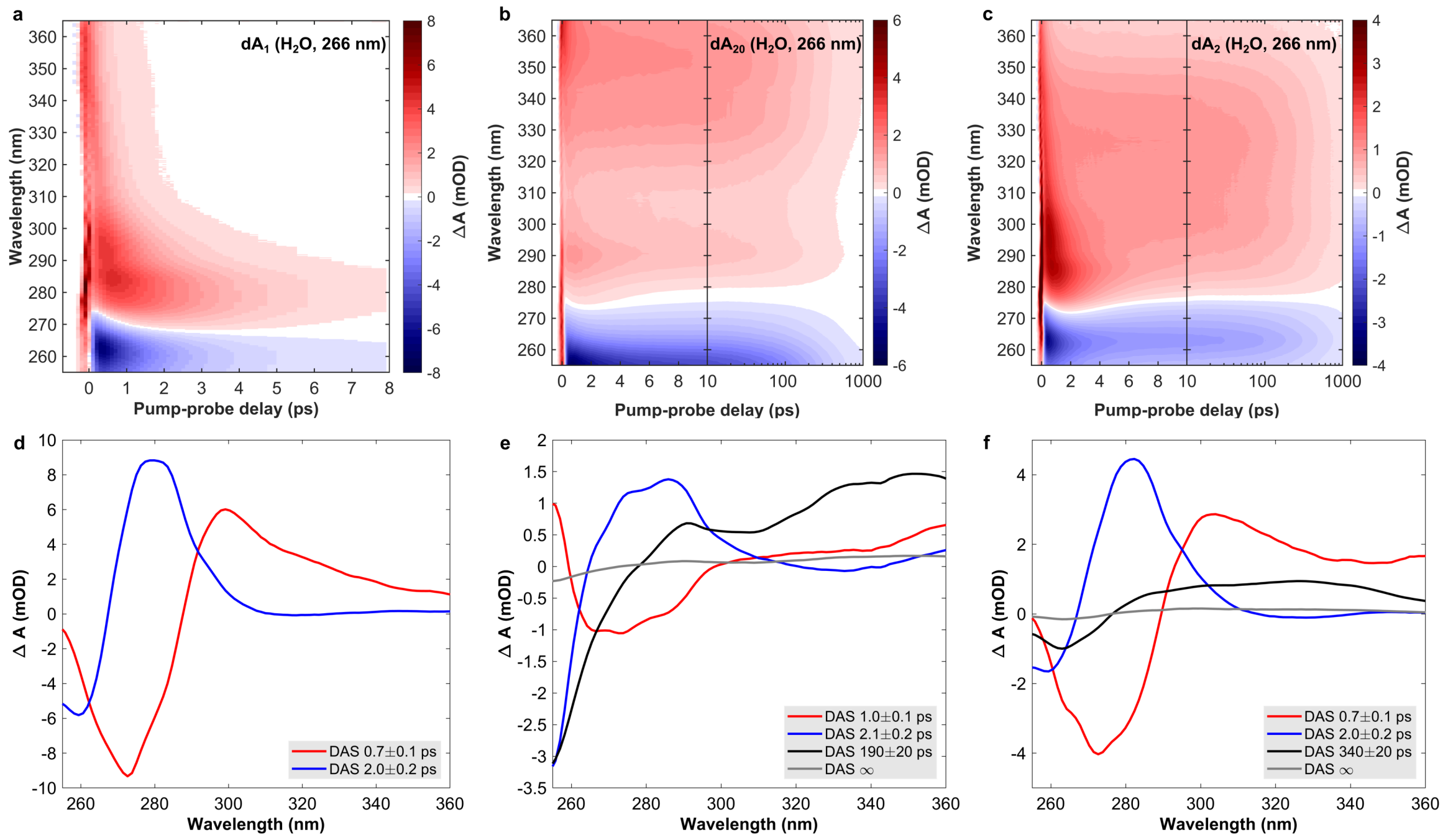

Figure S.4. a,b,c) Time-wavelength TA maps for $\mathrm{dA}_{1}, \mathrm{dA}_{20}$, and $\mathrm{dA}_{2}$, respectively, in $\mathrm{H}_{2} \mathrm{O}$ buffer solution, photoexcited at 266 nm. d,e,f) DAS obtained from global fits of the respective data sets $(a, b, c)$, where a sequence of multiexponential decays convoluted with the experiment's instrument response function was employed as the fit function. 

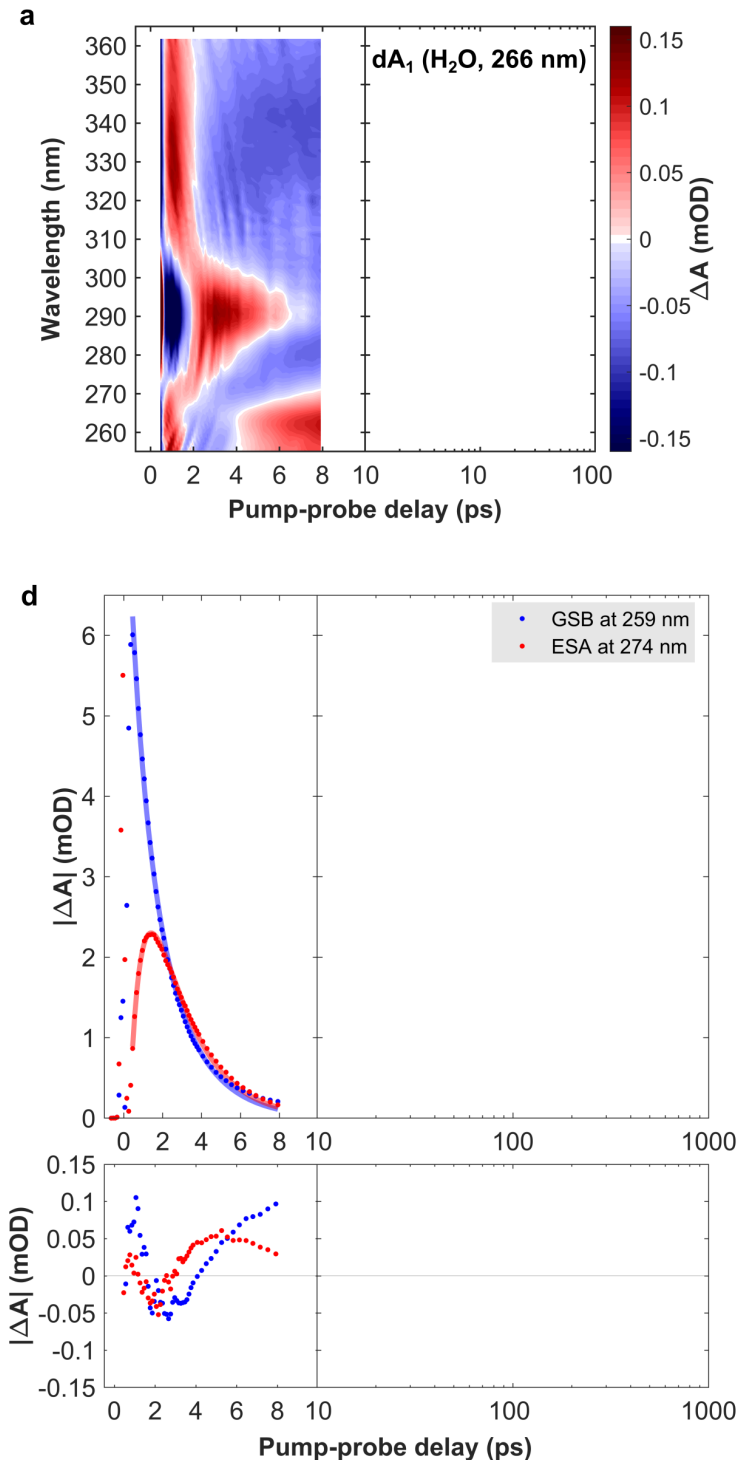

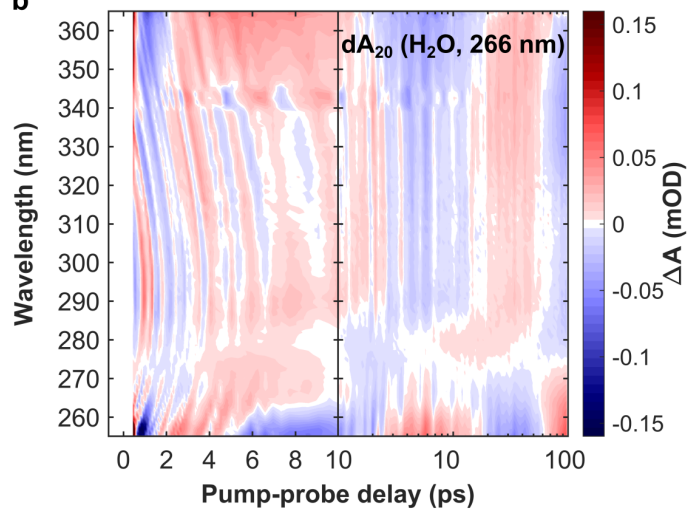

e $1+1,+\cdots$, GSB at $259 \mathrm{~nm}$
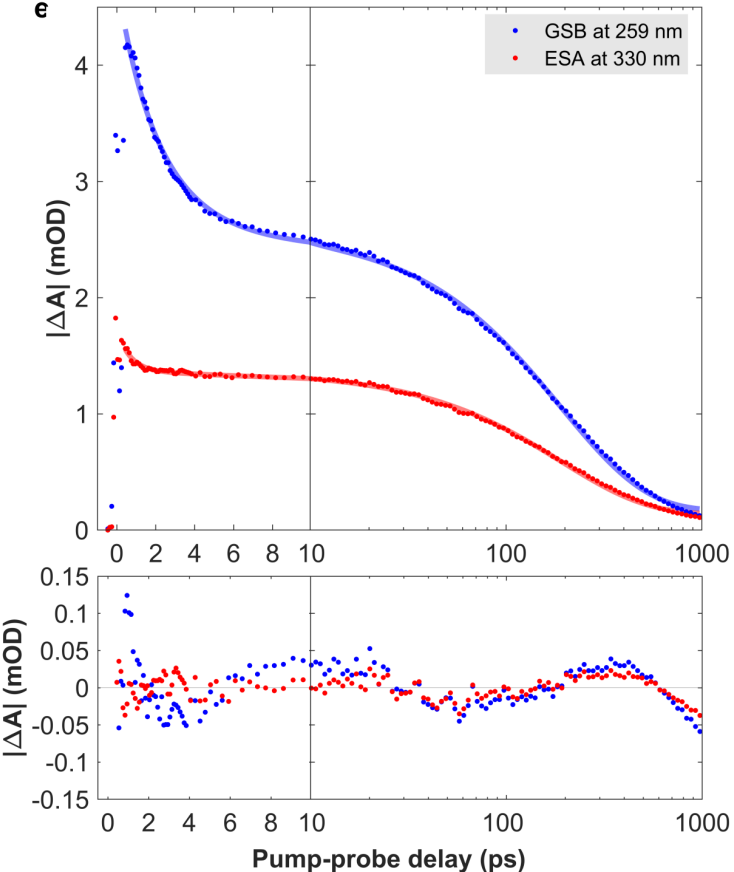
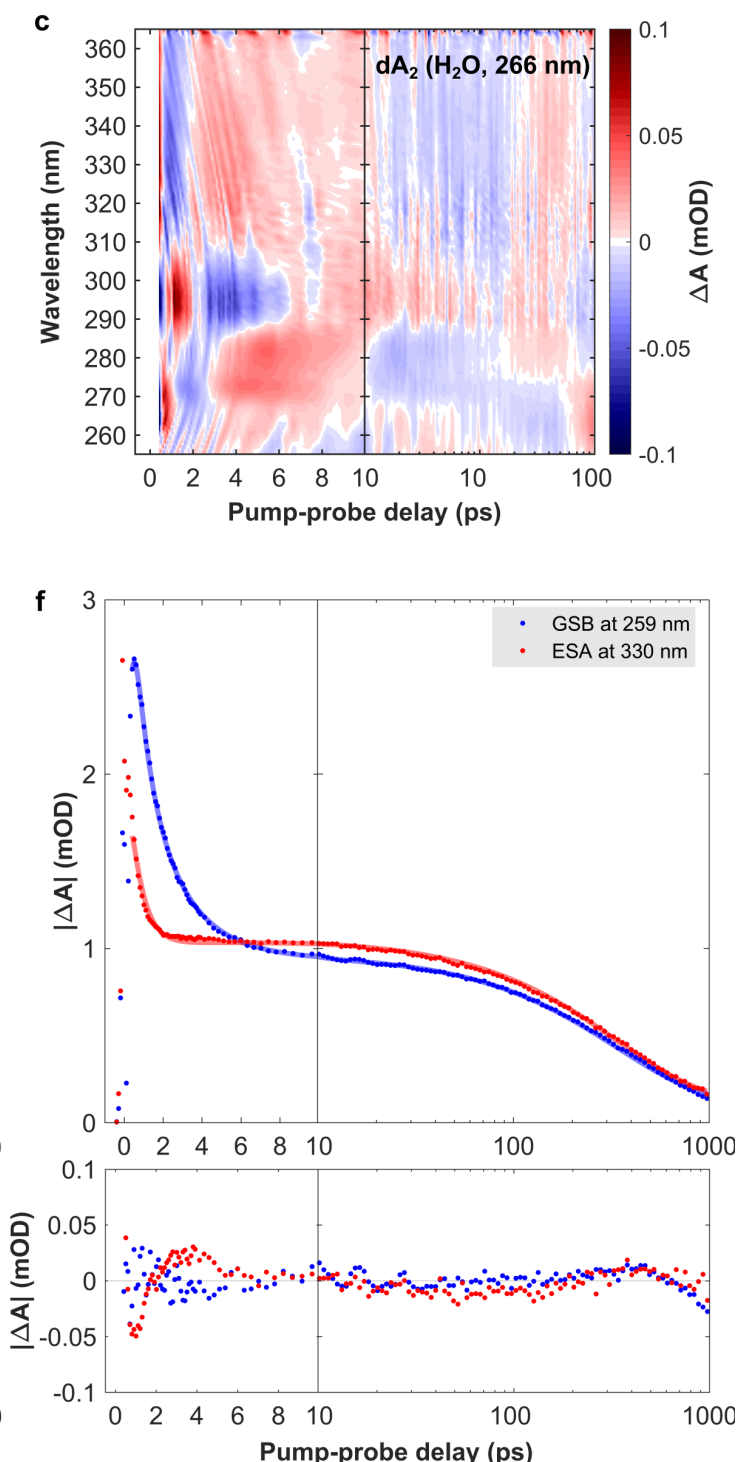

Figure S.5. a,b,c) Time-wavelength maps of the residuals obtained from the fits performed on the respective data sets displayed in fig. S.4. d,e,f) Absolute value of the TA amplitude as a function of pump-probe delay for selected probe wavelengths. Measured data points are displayed as dots and the multi-exponential fits as solid lines. The associated fit residuals are displayed in the lower panel. 

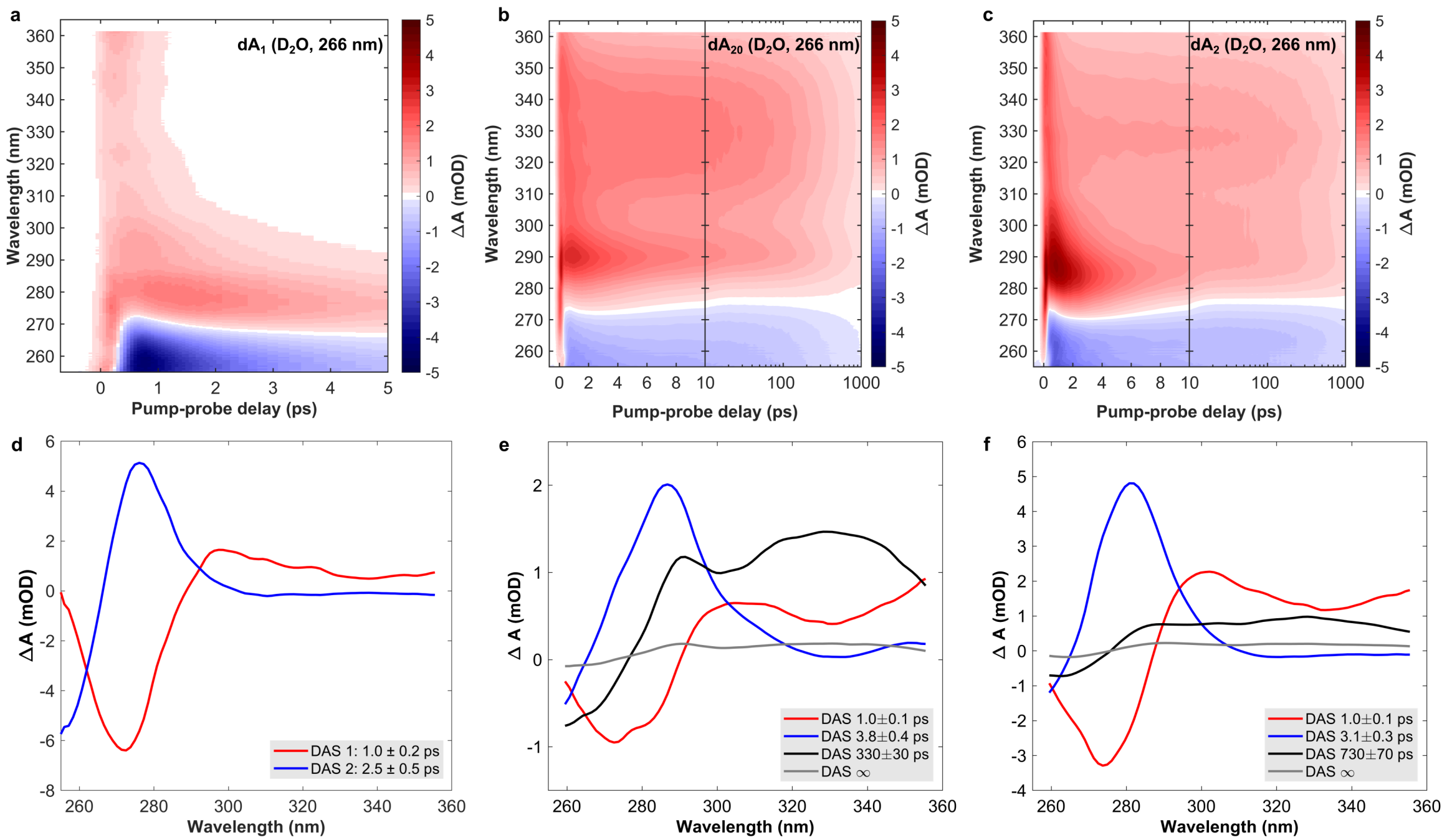

Figure S.6. a,b,c) Time-wavelength TA maps for $\mathrm{dA}_{1}, \mathrm{dA}_{20}$, and $\mathrm{dA}_{2}$, respectively, in $\mathrm{D}_{2} \mathrm{O}$ buffer solution, photoexcited at $266 \mathrm{~nm}$. d,e,f) DAS obtained from global fits of the respective data sets $(a, b, c)$, where a sequence of multiexponential decays convoluted with the experiment's instrument response function was employed as the fit function. 

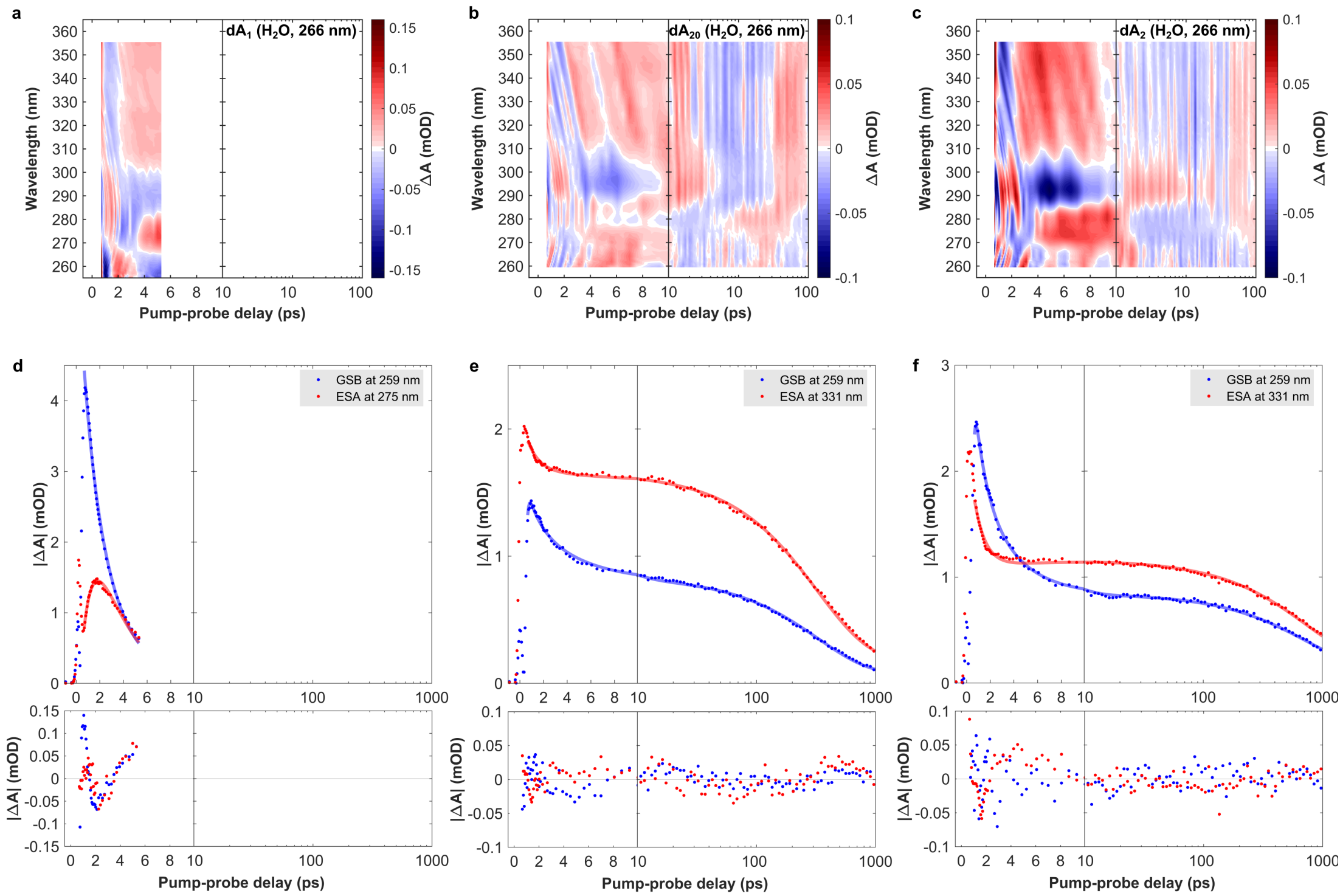

Figure S.7. a,b,c) Time-wavelength maps of the residuals obtained from the fits performed on the respective data sets displayed in fig. S.6. d,e,f) Absolute value of the TA amplitude as a function of pump-probe delay for selected probe wavelengths. Measured data points are 

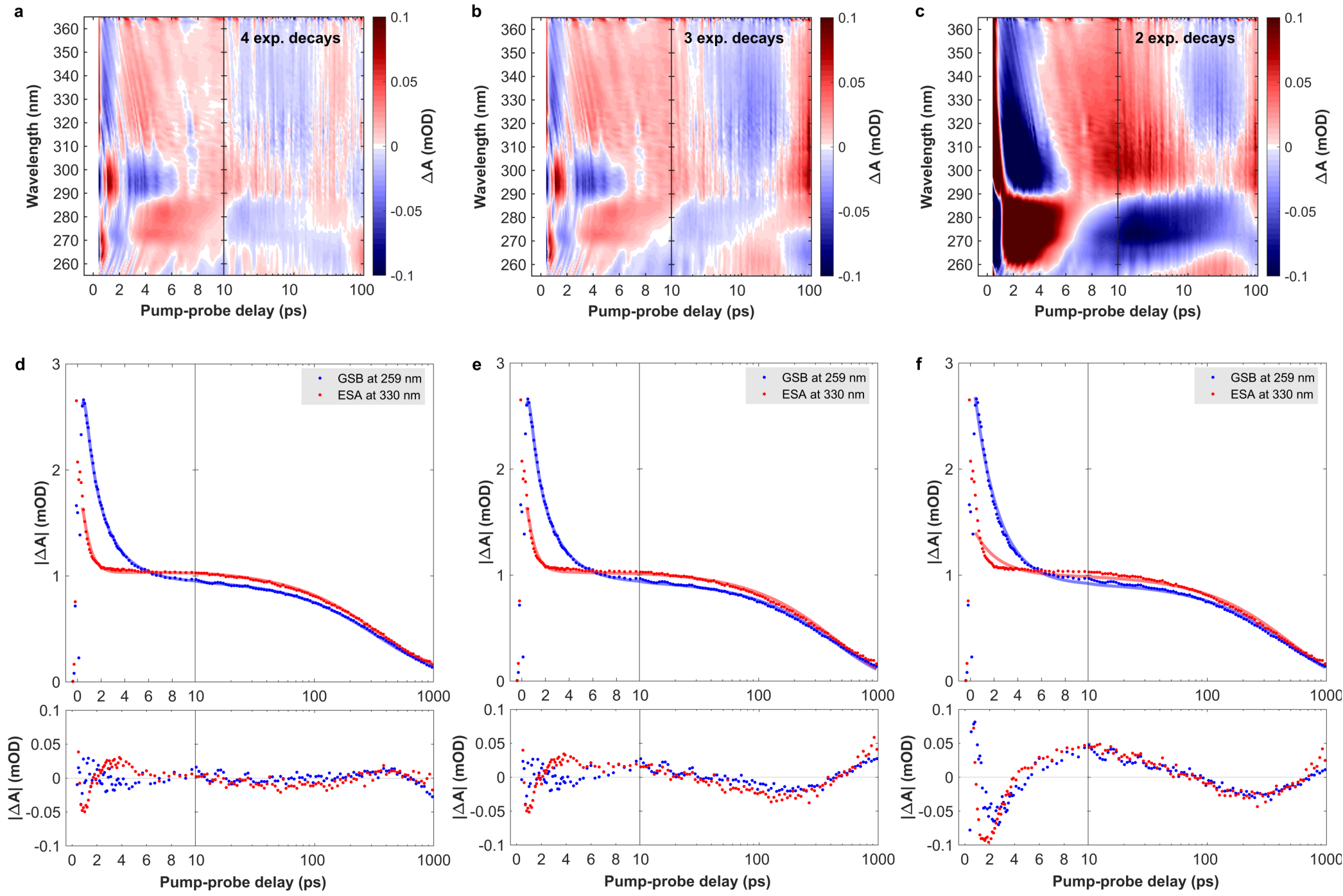

Figure S.8. a,b,c) Time-wavelength maps of the residuals obtained from multi-exponential fits performed on the TA data set for $\mathrm{dA}_{2}$ in $\mathrm{H}_{2} \mathrm{O}$ buffer solution, photoexcited at $266 \mathrm{~nm}$. To evaluate the number of exponential components required for an adequate fit, fit components were removed in a step-wise manner. Here the original fit with 4 components (a) is compared to a fit with 3 components (b), resulting in $\tau_{1} \approx 0.6 \mathrm{ps}, \tau_{2} \approx 2.1 \mathrm{ps}$, and $\tau_{3} \approx 460 \mathrm{ps}$, and 2 components (c), resulting in $\tau_{2} \approx 2.0 \mathrm{ps,}$ and $\tau_{3} \approx 490 \mathrm{ps}$. d,e,f) Absolute value of the TA amplitude as a function of pump-probe delay for selected probe wavelengths. Measured data points are displayed as dots and the multi-exponential fits as solid lines. The associated fit residuals are displayed in the lower panel. 

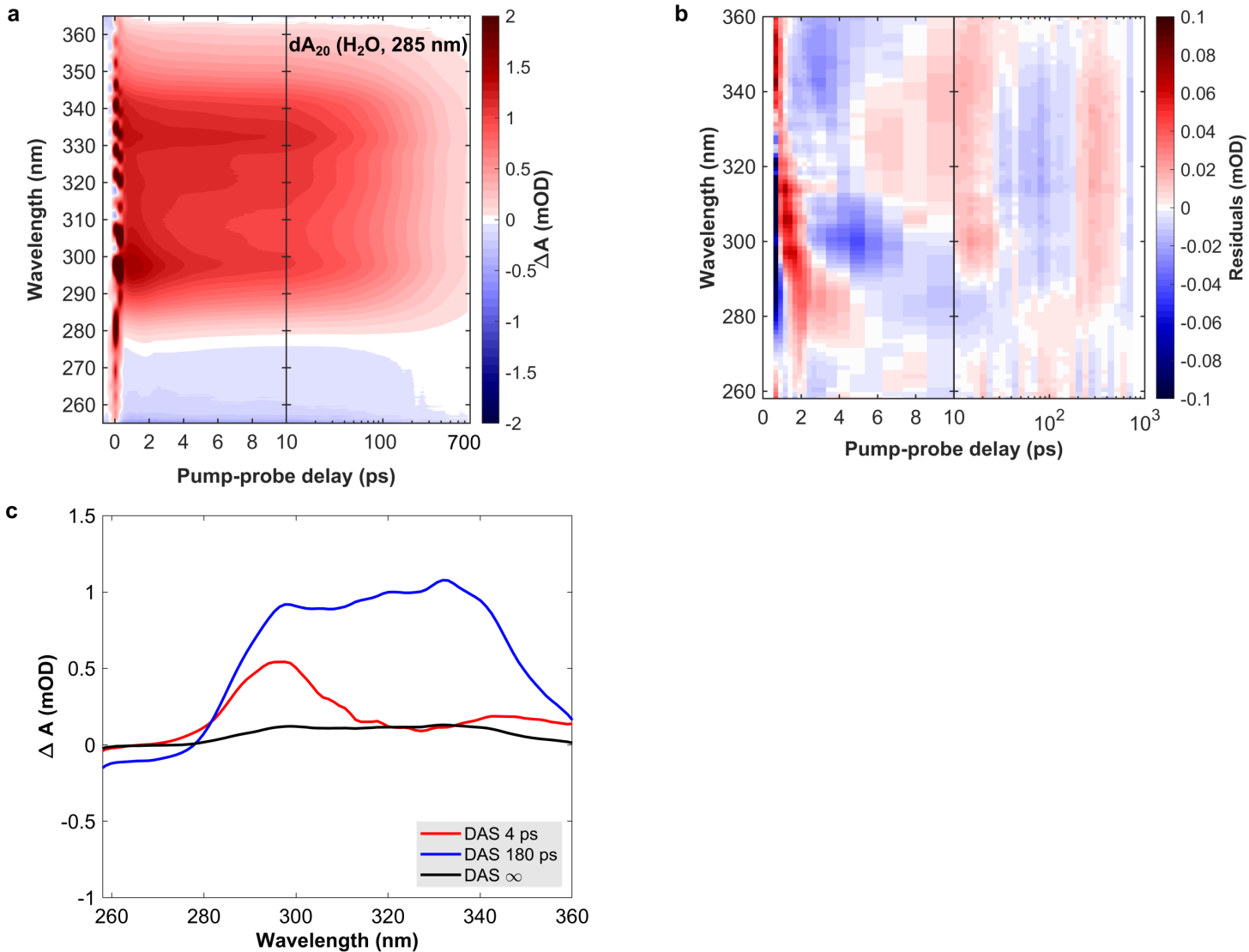

d
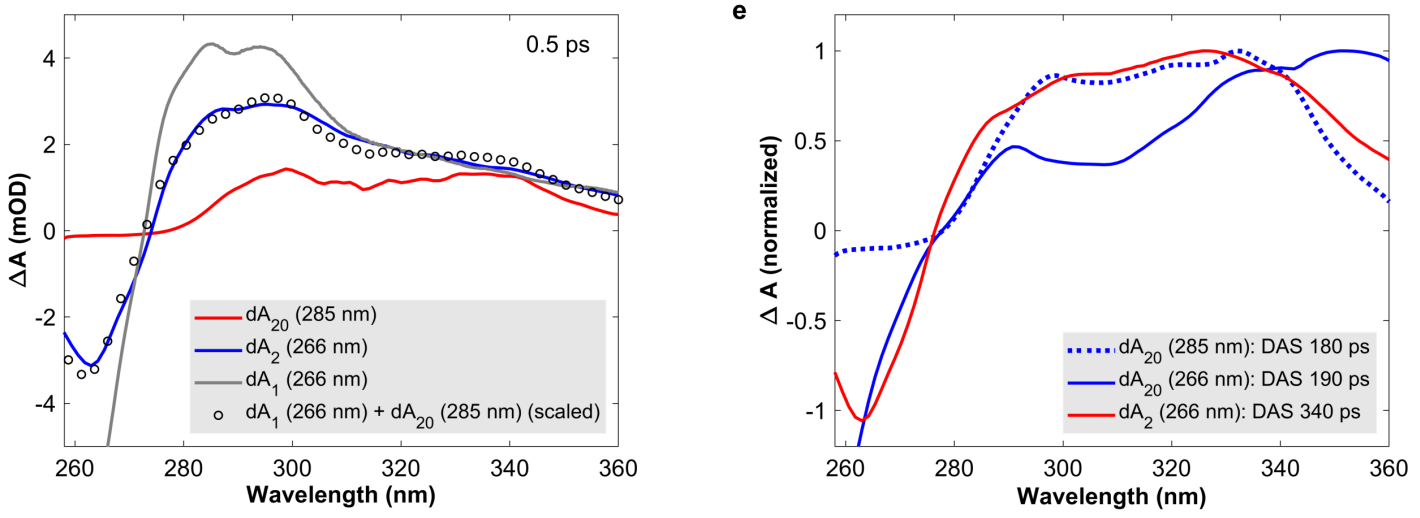

Figure S.9. a) Time-wavelength TA map for $\mathrm{dA}_{20}$ in $\mathrm{H}_{2} \mathrm{O}$ buffer solution, photoexcited at $285 \mathrm{~nm}$. b) Time-wavelength map of the residuals obtained from a global fit of data set (a), where a sequence of multiexponential decays convoluted with the experiment's instrument response function was employed as the fit function. c) DAS obtained from the global fit of data set (a). d) Comparison of the TA spectra at a pump-probe delay of $0.5 \mathrm{ps}$ for $\mathrm{dA}_{20}(285 \mathrm{~nm}), \mathrm{dA}_{2}(266 \mathrm{~nm})$, and $\mathrm{dA}_{1}(266 \mathrm{~nm})$ in $\mathrm{H}_{2} \mathrm{O}$ buffer solution. In addition the TA spectrum of $\mathrm{dA}_{2}(266 \mathrm{~nm})$ is compared to a sum of the TA spectra of $\mathrm{dA}_{20}(285 \mathrm{~nm})$ (multiplied by 0.75 ) and $\mathrm{dA}_{1}(266 \mathrm{~nm}$ ) (multiplied by 0.50$)$. The scaling factors were determined empirically. e) Comparison of the DAS associated with the CT exciton relaxation $\mathrm{dA}_{20}(285 \mathrm{~nm}), \mathrm{dA}_{20}$ (266 nm), and $\mathrm{dA}_{2}(266 \mathrm{~nm})$ in $\mathrm{H}_{2} \mathrm{O}$ buffer solution. Note that each DAS was normalized to its ESA maximum. 


\section{S.4 Transient absorption anisotropy}

As pointed out in the Methods section, the TAA data was obtained from the same experiments that resulted in the TA data sets presented previously. For the quantitative analysis of the TAA data sets the OPTIMUS software package was then used to perform global multi-exponential fits. As the calculation of the TAA spectra involves the TA spectrum as a denominator, the TAA amplitude diverges when the TA amplitude takes values close to zero. This is the case at probe wavelengths near zero-crossings between GSB and ESA bands, at early pump-probe delays due to cross-phase modulation (see section on the IRF determination) and at late pump-probe delays where the excited state population approaches zero. For the performed global fits we accounted for all three factors by excluding probe wavelengths near zero-crossings and by excluding pump-probe delays $<0.5 \mathrm{ps}$. Since each sample displayed different excited state lifetimes, the longest pump-probe delay included in the global fit was adapted accordingly. To this end, fig. S.10 displays the time-wavelength TAA maps for $\mathrm{dA}_{20}(266 \mathrm{~nm})$ and $\mathrm{A}_{2}(266 \mathrm{~nm})$ in $\mathrm{H}_{2} \mathrm{O}$ buffer solution, along with the time-wavelength maps of the residuals obtained from the global fits. The residual maps only display non-zero values in the regions that were included in the fits, thus illustrating the chosen boundaries for each sample. Fig. S.11 then displays the DAS obtained from the global fits, along with representative time traces of the TAA signal in the GSB and ESA regions of the TAA maps. For completeness, fig. S.15 displays the time-wavelength TAA maps for $\mathrm{dA}_{1}(266 \mathrm{~nm})$ in $\mathrm{H}_{2} \mathrm{O}$ and $\mathrm{D}_{2} \mathrm{O}$ buffer solution. We found that for $\mathrm{dA}_{20}(266 \mathrm{~nm})$ a minimum number of three exponential decays were required to fit the data. The high quality of the fit is illustrated by the low amplitude and uniform distribution of the residuals and by comparing the time trace data (dots) to the fitted curve (solid lines). Despite the good quality of the global fits, we estimated the errors of the resulting time constants to be larger than $20 \%$. The errors were obtained by systematically varying the boundaries of the time-wavelength TAA map included in the fit and observing the variation in the fit results. In the case of $\mathrm{dA}_{2}(266 \mathrm{~nm})$, we found that a minimum number of two exponential decays was required to achieve a good fit of the data. In this particular case, slight mismatches of the fitted curve with the data can be observed in fig. S.11d, suggesting the presence of an additional decay component. Whilst an additional decay with a time constant of approximately $50 \mathrm{ps}$ can be included in the fit, we decided against this approach upon review of all other TAA data sets involving $\mathrm{dA}_{2}$. Indeed, fitting three exponential decays to repeated experiments on $\mathrm{dA}_{2}(266 \mathrm{~nm})$ in $\mathrm{H}_{2} \mathrm{O}$ and $\mathrm{D}_{2} \mathrm{O}$ 
buffer solution did not converge to meaningful solutions in the majority of the obtained data sets.

Moving on, fig. S.12 and fig. S.13 display the TAA data sets and the results of their global analysis for $\mathrm{dA}_{20}(266 \mathrm{~nm})$ and $\mathrm{A}_{2}(266 \mathrm{~nm})$ in $\mathrm{D}_{2} \mathrm{O}$ buffer solution. Whilst the solvent deuteration shows a pronounced impact on the TA kinetics, a comparable effect is not observed in the TAA. Indeed, the obtained time constants mostly agree within their error ranges. Likewise, the quality of the fits is comparable to the experiments obtained in $\mathrm{H}_{2} \mathrm{O}$.

To check that the TAA data sets of $\mathrm{dA}_{20}$ in $\mathrm{H}_{2} \mathrm{O}$ and $\mathrm{D}_{2} \mathrm{O}$ buffer solution indeed require three exponential decay components, we performed global fits without the intermediate component $\rho_{2}$. The obtained time traces and fit residuals are displayed in fig. S.14 and show a pronounced mismatch between the data and the fit for pump-probe delays $>5$ ps. This confirms that three decay components are necessary to adequately fit the TAA data sets of the multimer samples.

\begin{tabular}{|l|l|l|}
\hline Sample & GSB & ESA \\
\hline $\mathrm{dA}_{1}, \mathrm{H}_{2} \mathrm{O}, 266 \mathrm{~nm}$ & $254-260 \mathrm{~nm}$ & $296-306 \mathrm{~nm}$ \\
\hline $\mathrm{dA}_{2}, \mathrm{H}_{2} \mathrm{O}, 266 \mathrm{~nm}$ & $255-267 \mathrm{~nm}$ & $310-340 \mathrm{~nm}$ \\
\hline $\mathrm{dA}_{20}, \mathrm{H}_{2} \mathrm{O}, 266 \mathrm{~nm}$ & $251-257 \mathrm{~nm}$ & $310-340 \mathrm{~nm}$ \\
\hline
\end{tabular}

Table S.1. Spectral boundaries for calculating the average TAA values in the GSB and ESA regions of the data sets for $\mathrm{dA}_{1}(266 \mathrm{~nm}), \mathrm{dA}_{2}(266 \mathrm{~nm})$, and $\mathrm{dA}_{2}(266 \mathrm{~nm})$ in $\mathrm{H}_{2} \mathrm{O}$ buffer solution. The resulting time traces are displayed in fig. $4 \mathrm{~b}$ in the main text. 

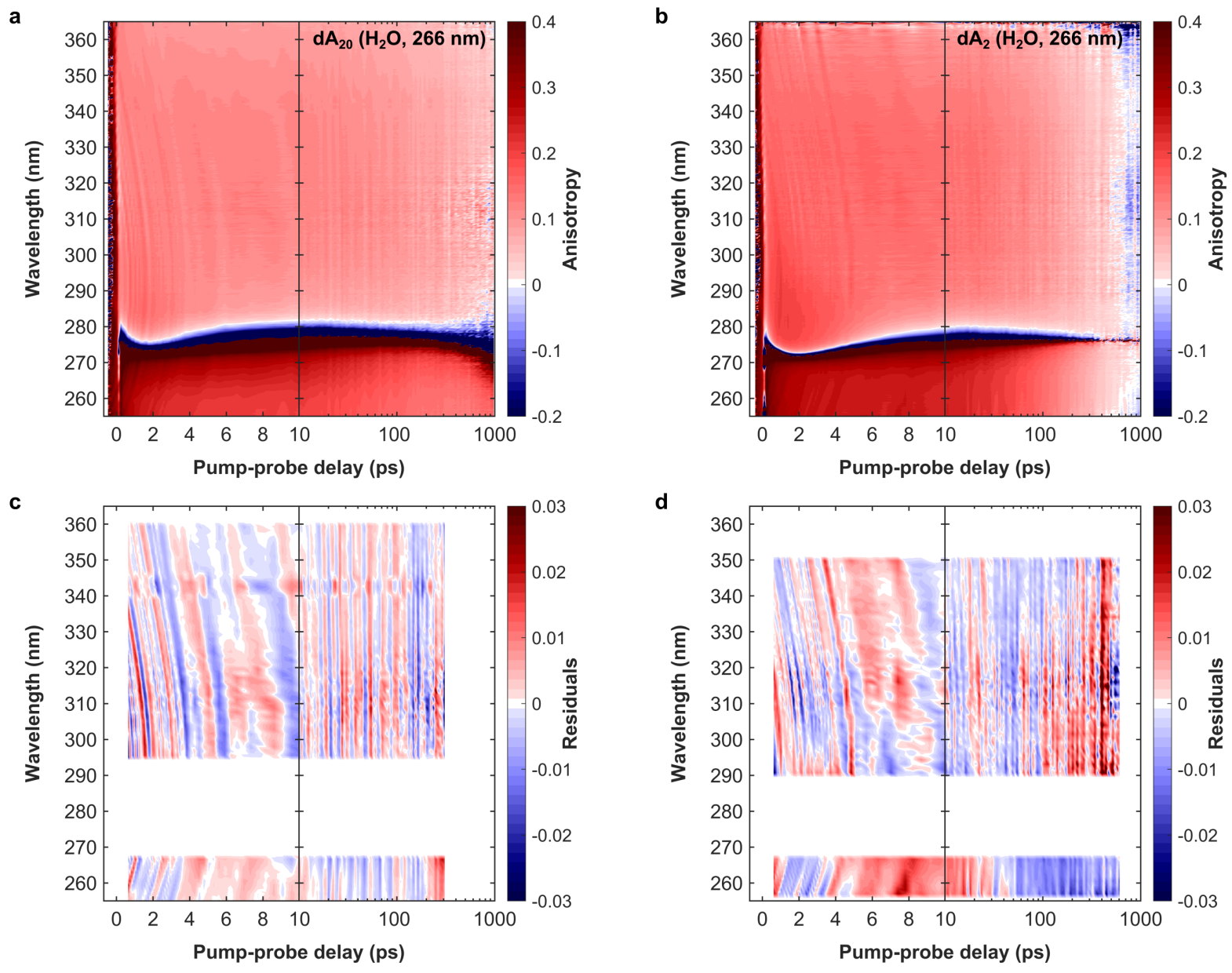

Figure S.10. a,b) Time-wavelength TAA maps for $\mathrm{dA}_{20}$ and $\mathrm{dA}_{2}$ in $\mathrm{H}_{2} \mathrm{O}$ buffer solution, photoexcited at $266 \mathrm{~nm}$. c,d) Time-wavelength maps of the residuals obtained from the global fits of the respective data sets $(a, b)$, with details explained in the text. 
a

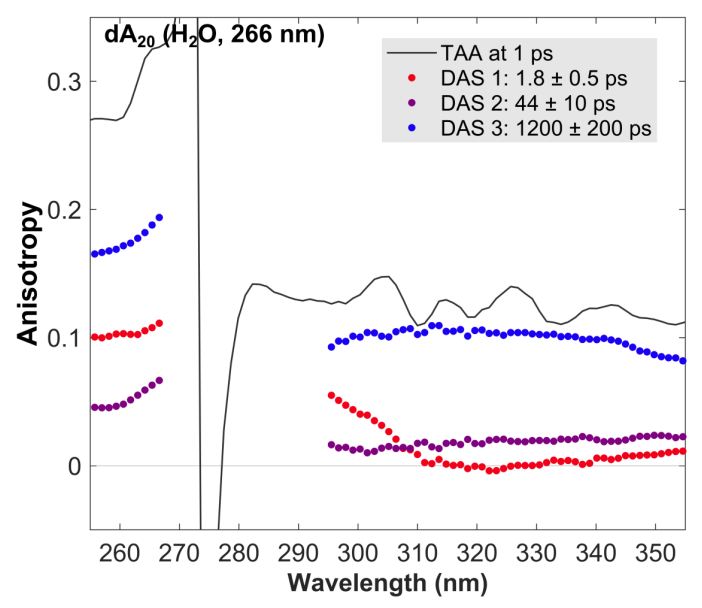

c

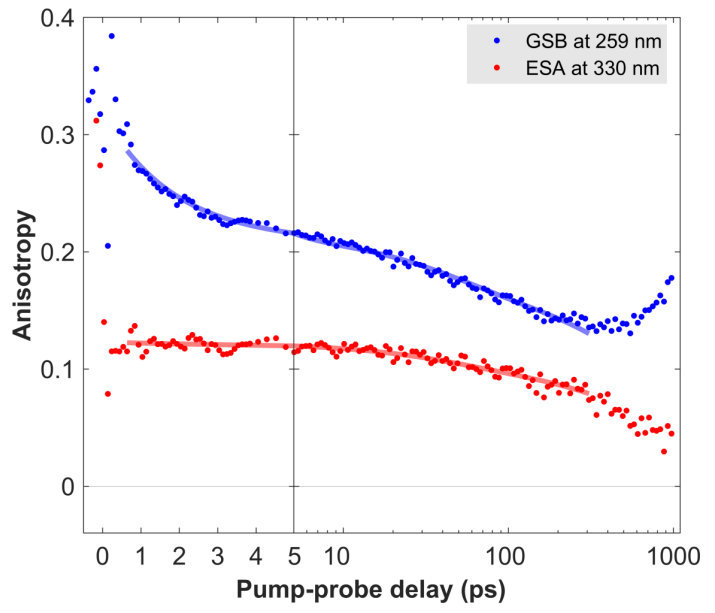

b

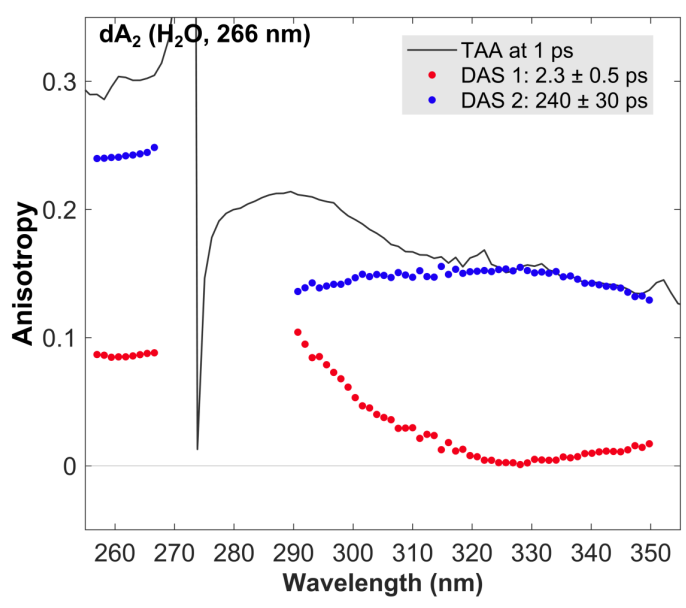

d

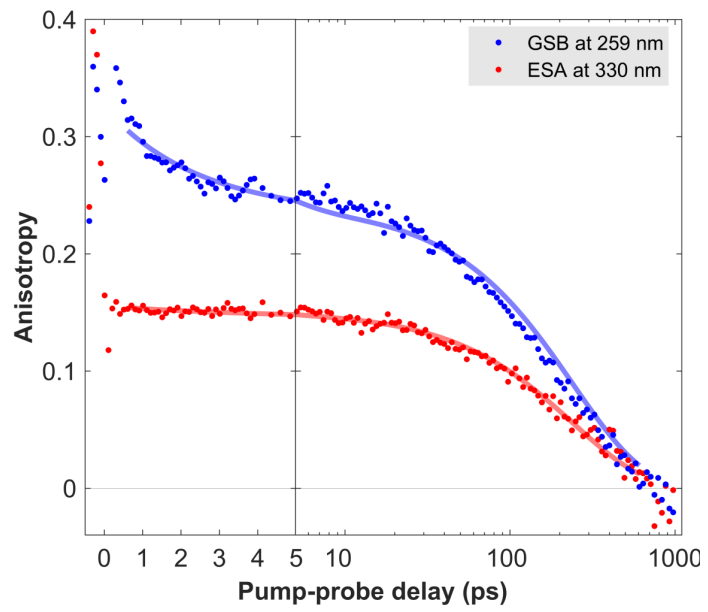

Figure S.11. a,b) DAS obtained from the global fits performed on the TAA data sets for $\mathrm{dA}_{20}(266 \mathrm{~nm})$ and $\mathrm{dA}_{2}(266 \mathrm{~nm})$ in $\mathrm{H}_{2} \mathrm{O}$ buffer solution. Note that a TAA spectrum at a pump-probe delay of $1 \mathrm{ps}$ is included in order to show the amplitude and spectral width of the TAA bands in the GSB and ESA regions. c,d) Time traces of the TAA data (dots) in the GSB and ESA region at the spectral positions indicated in the figure legend. The solid lines show the fitted curves obtained from the global fits for a visual comparison with the data. 

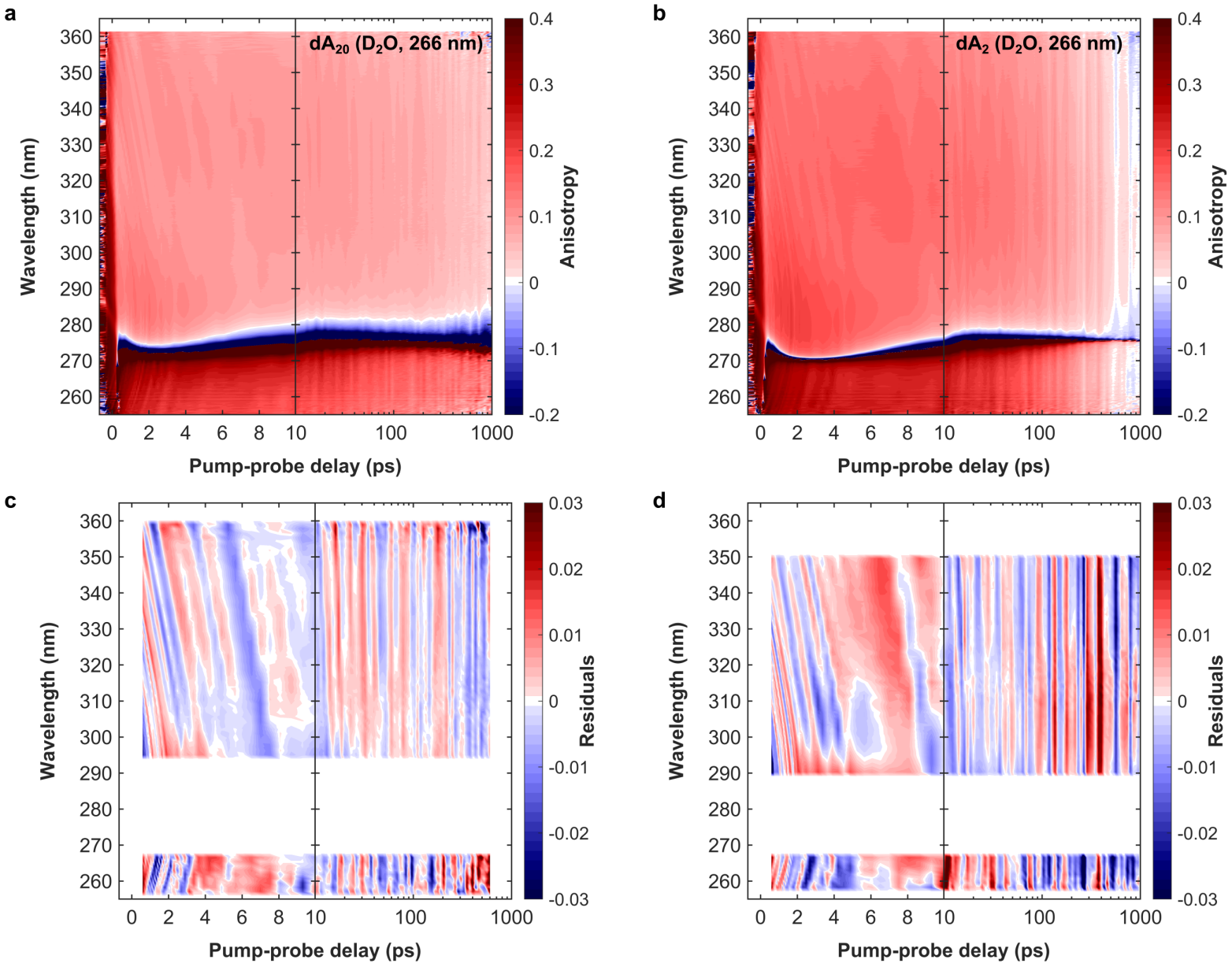

Figure S.12. a,b) Time-wavelength TAA maps for $\mathrm{dA}_{20}$ and $\mathrm{dA}_{2}$ in $\mathrm{D}_{2} \mathrm{O}$ buffer solution, photoexcited at $266 \mathrm{~nm}$. c,d) Time-wavelength maps of the residuals obtained from global fits of the respective data sets $(a, b)$, with details explained in the text. 
a

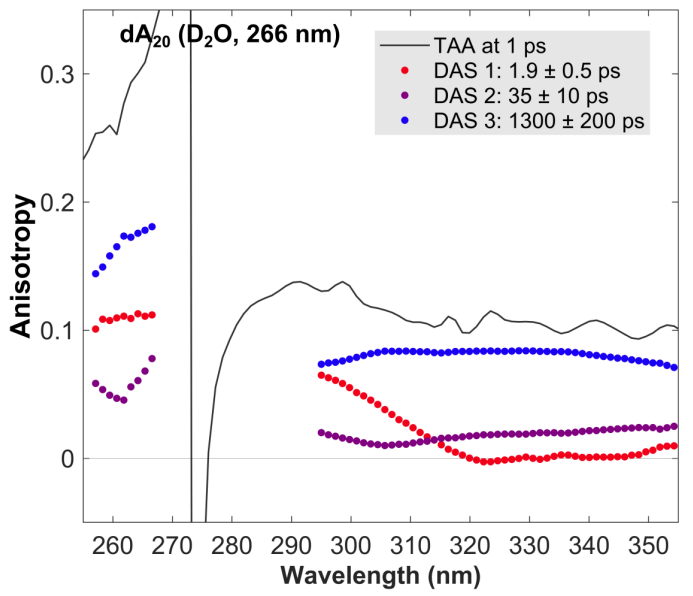

c

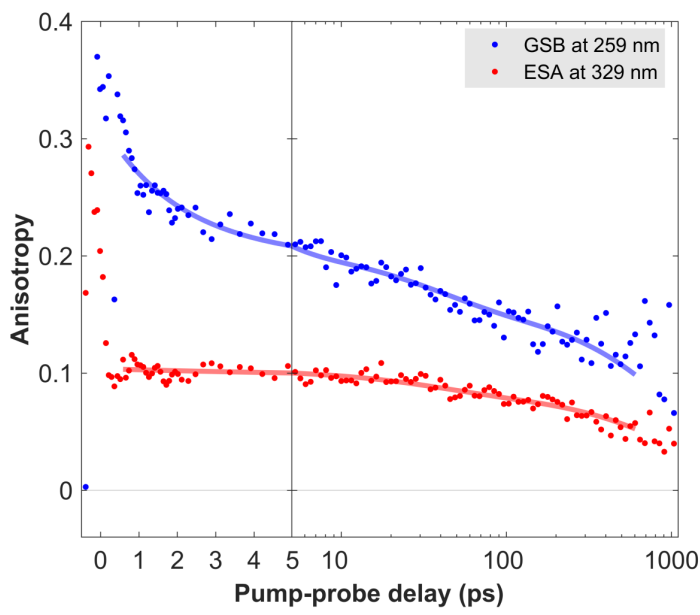

b

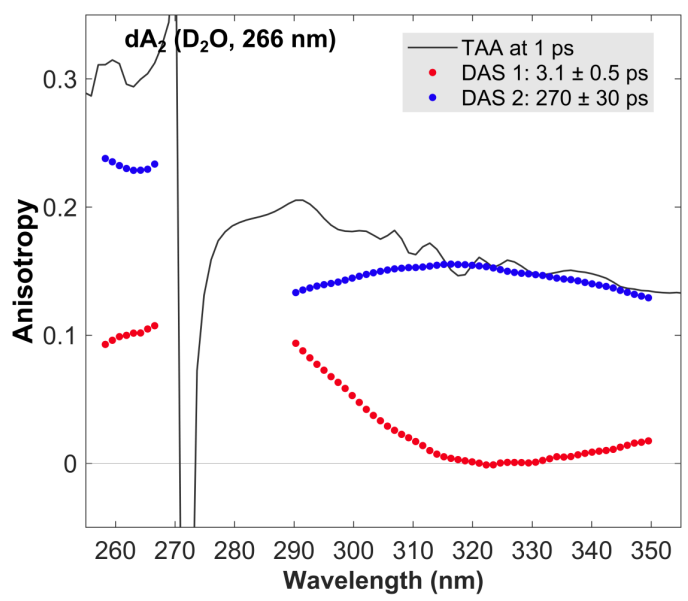

d

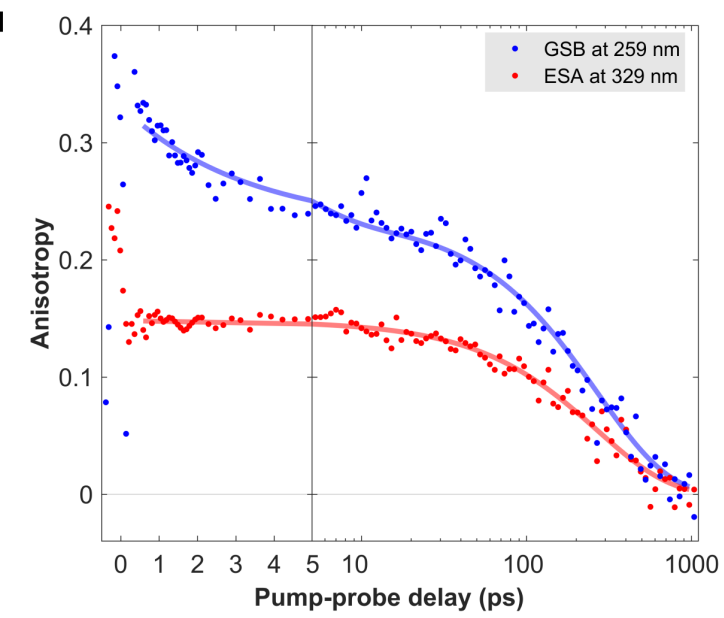

Figure S.13. a,b) DAS obtained from the global fits performed on the TAA data sets for $\mathrm{dA}_{20}(266 \mathrm{~nm})$ and $\mathrm{dA}_{2}(266 \mathrm{~nm})$ in $\mathrm{D}_{2} \mathrm{O}$ buffer solution. Note that a TAA spectrum at a pump-probe delay of $1 \mathrm{ps}$ is included in order to show the amplitude and spectral width of the TAA bands in the GSB and ESA regions. c,d) Time traces of the TAA data (dots) in the GSB and ESA region at the spectral positions indicated in the figure legend. The solid lines show the fitted curves obtained from the global fits for a visual comparison with the data. 
a

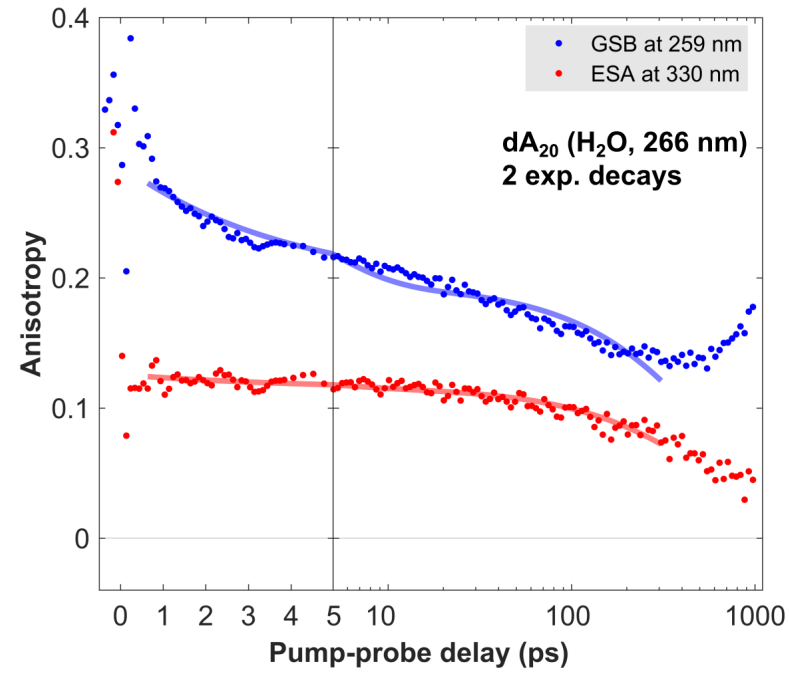

c

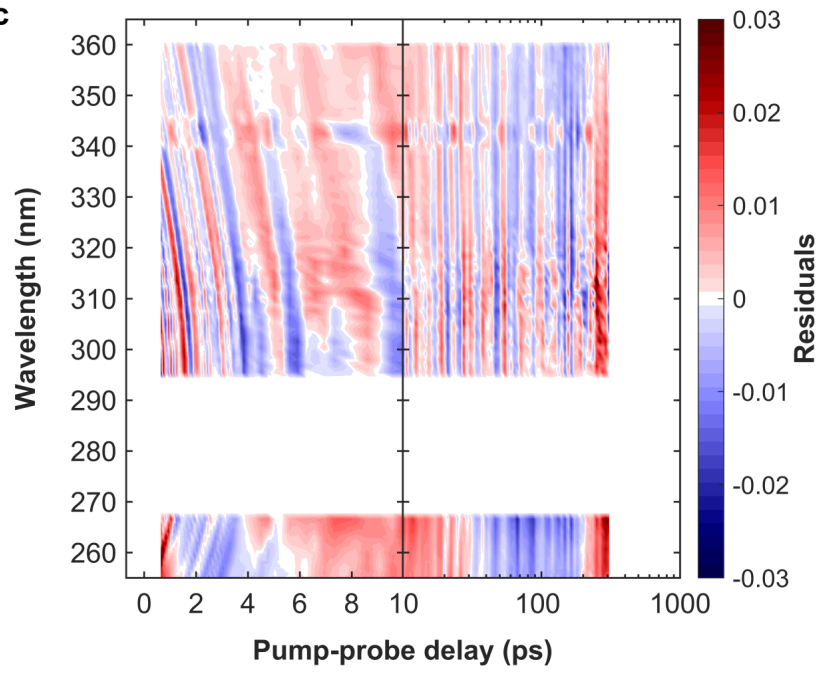

b
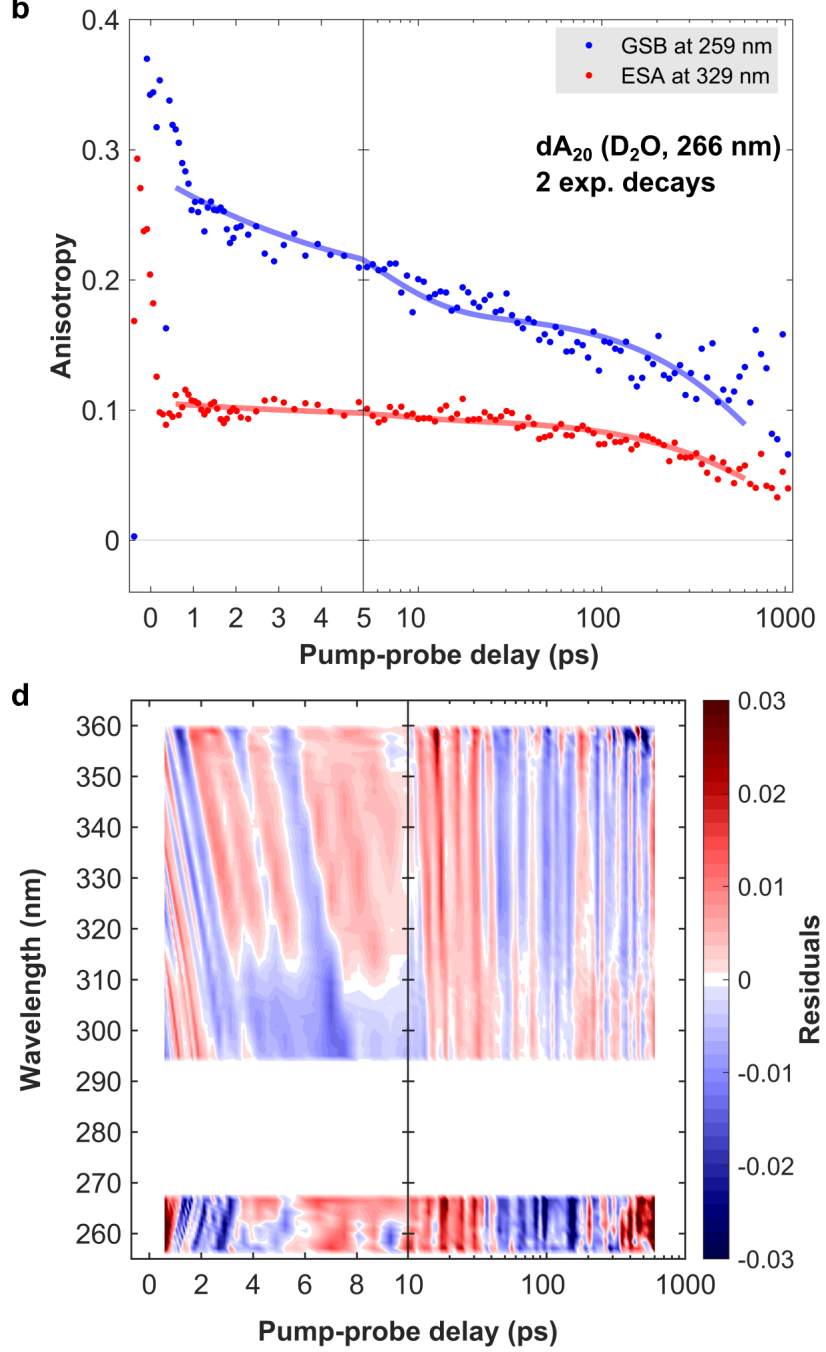

Figure S.14. Evaluation of the number of fit components required for the TAA data sets of $\mathrm{dA}_{20}$ (266 $\mathrm{nm}$ ) in $\mathrm{H}_{2} \mathrm{O}$ (left panels) and $\mathrm{D}_{2} \mathrm{O}$ buffer solution (right panels). In both cases the intermediate decay component $\rho_{2}$ was removed from the global fit. Time traces of the TAA data (dots) in the GSB and ESA region at the spectral positions indicated in the figure legend. The solid lines show the fitted curves obtained from the global fits for a visual comparison with the data. The obtained decay constants are $\rho_{1} \approx 3.8$ ps and $\rho_{3} \approx 650$ ps in $\mathrm{H}_{2} \mathrm{O}$ (a) and $\rho_{1} \approx 5.3$ ps and $\rho_{3} \approx 890$ ps in $\mathrm{D}_{2} \mathrm{O}$ (b). c,d)

Time-wavelength maps of the residuals obtained from the global fits of the respective data sets $(a, b)$ 

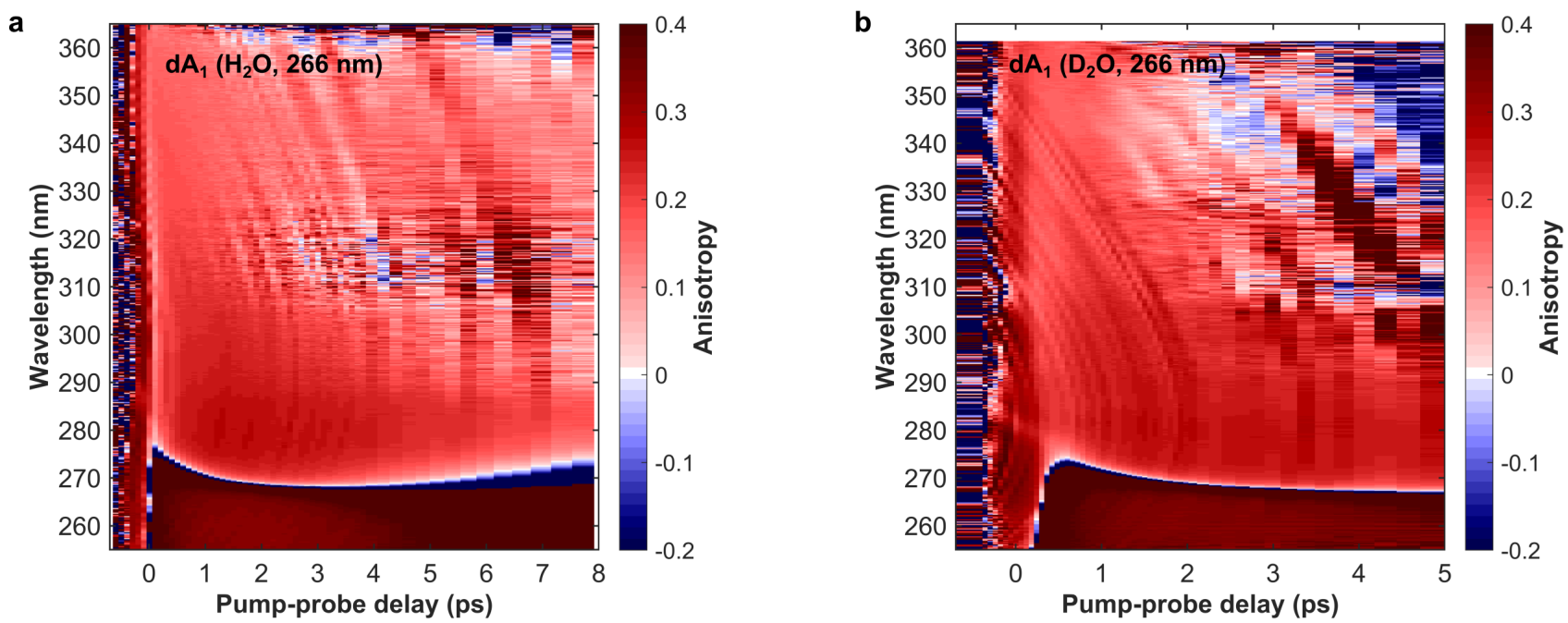

Figure S.15. a,b) Time-wavelength TAA maps for $\mathrm{dA}_{1}$ in $\mathrm{H}_{2} \mathrm{O}$ and $\mathrm{D}_{2} \mathrm{O}$ buffer solution, photoexcited at $266 \mathrm{~nm}$. 